TTK-11-63

\title{
Effective Theory of Resonant Leptogenesis in the Closed-Time-Path Approach
}

\author{
Björn Garbrecht and Matti Herranen* \\ Institut für Theoretische Teilchenphysik und Kosmologie, \\ RWTH Aachen University, 52056 Aachen, Germany
}

\begin{abstract}
We describe mixing scalar particles and Majorana fermions using Closed-TimePath methods. From the Kadanoff-Baym equations, we obtain the charge asymmetry, that is generated from decays and inverse decays of the mixing particles. Within one single formalism, we thereby treat Leptogenesis from oscillations and recover as well the standard results for the asymmetry in Resonant Leptogenesis, which apply when the oscillation frequency is much larger than the decay rate. Analytic solutions for two mixing neutral particles in a constant-temperature background illustrate our results qualitatively. We also perform the modification of the kinetic equations that is necessary in order to take account of the expansion of the Universe and the washout of the asymmetry.
\end{abstract}

\footnotetext{
*Alexander-von-Humboldt Fellow
} 


\section{Introduction}

There are several different methods of calculating the lepton asymmetry, that may be generated in the Early Universe. This variety of approaches is a feature that Leptogenesis [1] has in common with many other topics in Particle Theory.

The approach that is perhaps most commonly applied is to calculate the decay asymmetry of individual right handed, singlet Majorana neutrinos $N$ from $S$-matrix elements [1 4]. These asymmetries, obtained by Quantum Field Theoretical methods, are subsequently substituted into classical Boltzmann equations, that describe the macroscopic non-equilibrium dynamics and the evolution of the asymmetry. By LSZ-reduction, $S$-matrix elements can be obtained from time ordered $n$-point Green functions. We then enjoy the usual simplifications of time-ordered perturbation theory, i.e. we can calculate the $n$-point functions using the standard diagrammatic Feynman rules. The price to pay for this simplification is cutting the direct link between the non-equilibrium dynamics and the generation of the $C P$-asymmetry at the quantum level. A particular problem turns out to be due to the instability of the singlet neutrinos, which causes that the $S$-matrix, that involves the $C P$-asymmetric decays and inverse decays of these singlets, is not unitary, or rather, that it does not correspond to an $S$-matrix in the proper sense. As a consequence, the unitary evolution of the non-equilibrium system must be carefully re-implemented, which is performed in practise through the non-trivial method of real intermediate state subtraction [5]. Without real intermediate state subtraction, one would predict from substituting the rates from the $S$-matrix elements into the network of Boltzmann equations the presence of a lepton asymmetry even in thermal equilibrium, which would be an unacceptable violation of the CPT theorem.

For Resonant Leptogenesis [3, 6 - 10], it is assumed that the mass difference $\Delta M$ of two of the singlet neutrinos is small compared to their average mass $\bar{M}$, or, more precisely, the energy difference is small compared to the average energy. Decays and inverse decays, that induce off-diagonal correlations of the singlet neutrinos in their mass eigenbasis, then lead to a relatively large mixing, which enhances $C P$-violation. In the $S$-matrix approach, the mixing appears as a wave function correction to the singlet neutrino propagator in the decay diagrams [3, 11 13]. The mixing that is calculated in this way is time-independent, which excludes the possibility of incorporating the effect of oscillations.

Alternatively, one can avoid the subtleties concerning unitarity by computing the real-time evolution of the quantum state from the outset. This can either be achieved by using the canonical Hamiltonian approach or functional, Closed-Time-Path (CTP) [1417. methods. This may appear as a complicated task, but one should keep in mind that the $C P$-asymmetry arises in the $S$-matrix language at the one-loop level. In a real time approach, it turns out that in a diagrammatic representation, we have to go to two-loop level. However, the leading $C P$-violating effects can be extracted by imposing some of the internal particles to be on-shell, which may be considered as a generalisation of the Cutkosky rules [18]. In the end, the integrals that are relevant for the leading order prediction of the $C P$ asymmetry are identical to those encountered in the $S$-matrix 
approach, but with the advantage that an overcounting of particular processes in the Boltzmann equations, that would violate unitarity, is avoided by construction.

The canonical approach makes use of the property of the Hamiltonian as the time evolution operator. A blueprint of this canonical approach, is the theory of neutrino oscillations in Refs. 19 23. It has been noticed that when supplemented by $C P$-violation, the oscillations may lead to the creation of a lepton asymmetry [24 26]. The possibility of describing mixing oscillations is one key advantage of the canonical approach when compared to using $S$-matrix elements. On the other hand, without a set of diagrammatic rules, the perturbation expansion is rather cumbersome. This is why the remaining important $C P$-violating contribution, that is sometimes referred to as $\operatorname{direct} C P$-violation (e.g. in Ref. [6]), has not been calculated in the Hamiltonian approach. In the $S$-matrix approach, this contribution arises from the penguin-shaped vertex diagram. Besides, the decays of the singlet neutrinos into Standard Model leptons are described in practise by appealing again to the $S$-matrix formalism. Therefore, one may state that within the Hamiltonian approach, no entirely closed picture for the emergence of the lepton asymmetry has yet been developed.

In principle, it should be possible to derive a set of diagrammatic rules that simplifies the calculations within the Hamiltonian formalism. However, such a set of rules is readily available. It arises in an elegant and rather intuitive manner from the functional formulation of the CTP formalism [14 17]. In the CTP approach, the Hamiltonian time evolution of the density matrix is replaced by Schwinger-Dyson equations for the two-point Green functions. These equations have four matrix components from the two indices that denote the two branches of the CTP. The Kadanoff-Baym equations are those of the Schwinger-Dyson equations, that encompass the macroscopic evolution of the system. Recently, this approach has proved successful in order to describe Leptogenesis within a single theoretical framework [27 37]. Both, $C P$-violation from wave-function and vertex corrections are incorporated. Unitarity issues are resolved and an accurate account of all quantum-statistical effects on the asymmetry is made. Moreover, the formulation in terms of Green functions bears the potential of incorporating corrections from Thermal Field Theory within this formalism. For the $C P$-conserving production rate of singlet neutrinos, several phenomenologically relevant thermal corrections have recently been calculated in Refs. 38 44]. When it comes to the rate of $C P$-violation, an aspect that is not dealt with in Refs. [27 37] is the possibility of flavour oscillations between the singlet neutrinos. The present work addresses this shortcoming.

While in Ref. [33], the wave function correction is calculated using the perturbative loop expansion, the present work captures the same effect by solving the Kadanoff-Baym equations for the singlet neutrino propagator directly. In the limit where the mass difference is much larger than the decay rate $\Gamma_{\mathrm{D}}$ of the singlets, $\Delta M \gg \Gamma_{\mathrm{D}}$, the standard perturbative result is reproduced. When $\Delta M \sim \Gamma_{\mathrm{D}}$ or smaller, it becomes important that the solution to the Kadanoff-Baym equations corresponds to a resummation of all wave-function insertions. This is a generalisation of the familiar procedure for timeindependent situations, which can be performed by the summation of a geometric series. The result obtained in the present work from the Kadanoff-Baym equations encompasses 
both, time-independent and oscillating contributions to the mixing of the singlet neutrinos. We note that for a model of the asymmetric decay of a scalar field, it was clarified in Refs. [45, 46] that the Hamiltonian approach incorporates indeed the wave-function contribution to the $C P$-asymmetry from decays in the vacuum as it is usually calculated from $S$-matrix elements. The present work goes beyond this proof of principle not only because it also treats the decays of Majorana fermions, but also because it formulates the evolution of the asymmetry in an interacting, finite-density system within the single framework of the CTP formalism. Our results may therefore be employed for phenomenological studies of Resonant Leptogenesis in the Early Universe.

In order to further describe the context of this present work, we note that for computations of the asymmetry from $S$-matrix elements, it has been realised that the resonant enhancement factor $1 / \Delta M$ should be regulated by the finite width of the singlet neutrinos [4, 7 -10, 47]. For solutions to the Kadanoff-Baym equations in Wigner space, 1 it turns out that the non-equilibrium distribution functions are not multiplied by a finitewidth, Breit-Wigner distribution, but rather by a zero width, Dirac $\delta$-function. The observation of this somewhat curious property has lead to the speculation that these zero-width solutions arise due to an incorrect treatment of pinch singularities that must be addressed by a non-perturbative resummation [48, 49], that is yet to be specified. This may be perceived as an obstacle for the formulation of an effective theory of Resonant Leptogenesis within the CTP framework. However, it was recently demonstrated analytically and numerically, that the vanishing width is a correct property of the outof-equilibrium Wigner functions. Within loop diagrams, the zero width is corrected for by a resummation of derivative operators, which effectively shifts the argument of the $\delta$ function from the real axis into the imaginary direction of the complex plane [50]. In the Kadanoff-Baym equations, this effect may be of relevance when the tree-level scattering processes are kinematically forbidden. As this is not the situation that we assume for the present purposes, the corrections that are explained in Ref. [50] are subdominant in the final result for the asymmetry and therefore are neglected here. The results of Ref. [50] imply however, that a straightforward solution of the Kadanoff-Baym equations should indeed regulate the asymmetry when $\Delta M \rightarrow 0$. Indeed, this is what we explicitly find in the present work.

After these comments, we present the outline of this paper: In Section 2, we use the Schwinger-Dyson equations on the CTP, in particular the Kadanoff-Baym equations, in order to calculate the asymmetry that emerges from the decays of mixing neutral scalar particles. The Kadanoff-Baym equations decompose into constraint and kinetic equations. When $\Delta M \gg \Gamma_{\mathrm{D}}$, one may use either of these equations in order to derive the asymmetry. If this is not the case, one should use an expansion based on $\Delta M \ll$ $\bar{M}$. In this limit, the kinetic equations take the form that is familiar from the timeevolution of a density matrix in the Hamiltonian approach. In order to gain a qualitative understanding, we derive analytical solutions for the mixing propagator in the case of two mixing flavours in a constant-temperature background.

\footnotetext{
${ }^{1}$ The Wigner transform is the Fourier transform of a two-point function with respect to the relative coordinate, while retaining the average coordinate, see Appendix $\mathrm{A}$.
} 
In Section 3, we apply the same approximation strategies as for scalars to mixing Majorana neutrinos. As an additional complication, we need to solve for the various spinor components of the singlet neutrino propagator. However, it turns out that the flavour dynamics shares the same essential features with the scalar model. Even though our main interest is the decay of Majorana fermions, a reader who is less interested in these technical details can skip this Section and continue with Section 4. There, we discuss some features of the analytical solutions for the asymmetry in a model in constant temperature background, for both, fermions and scalars. In particular, we see that when $\Delta M \gg \Gamma_{\mathrm{D}}$, the effect of oscillations of the singlet neutrinos averages out and the standard results for the decay asymmetry from $S$-matrix elements apply. However, when the condition $\Delta M \gg \Gamma_{\mathrm{D}}$ does not apply, the oscillations can have a significant effect on the asymmetry.

In Section 5, we present the modifications of the kinetic equations that arise in an expanding Universe. We therefore eventually arrive at an effective theory for Resonant Leptogenesis, that is formulated within the CTP framework. In Section 6, there are a short summary of this paper as well as concluding remarks.

\section{Scalar Model}

\subsection{CTP Approach to Generating a Charge Asymmetry from out-of-Equilibrium Decays}

The perhaps simplest model for generating a charge asymmetry from out-of-equilibrium decays is given by $[29,30,46]$

$$
\mathcal{L}=\left(\partial_{\mu} \varphi\right)\left(\partial^{\mu} \varphi^{*}\right)-M_{\varphi}^{2}|\varphi|^{2}+\frac{1}{2}\left(\partial_{\mu} \chi_{i}\right)\left(\partial^{\mu} \chi_{i}\right)-\frac{1}{2} M_{\chi_{i j}}^{2} \chi_{i} \chi_{j}-g_{i} \chi_{i} \varphi^{2}-g_{i}^{*} \chi_{i} \varphi^{* 2} .
$$

The field $\varphi$ is a complex scalar that can be associated with a charge, while the $\chi_{i}$ are real singlet scalar fields, that can create a charge asymmetry within $\varphi$ when decaying out-of equilibrium. We sum over repeated indices $i, j$. We take here $i=1,2$, for simplicity. The generalisation to more than two flavours of $\chi$ is mostly straightforward, except for the analytic formulae involving the diagonalisation of matrices that we present in Section 2.3, We choose a basis where the mass-matrix $M_{\chi}$ is diagonal. The couplings $g_{i}$ are complex and of mass dimension one.

When replacing the $g_{i}$ by Yukawa couplings and the complex scalar $\varphi$ by a Higgs and a lepton field - which is straightforward to implement, above model describes soft Leptogenesis from sneutrino mixing [64 66]. This may be an interesting phenomenological application of the methods developed in the present work.

In Wigner space, the Kadanoff-Baym equations for the Green functions of the fields 
$\chi_{i}$ and $\varphi$ are

$$
\begin{gathered}
{\left[k^{2}-\frac{1}{4} \partial_{t}^{2}+\mathrm{i} k^{0} \partial_{t}-M^{2}\right] \Delta^{<,>}-\mathrm{e}^{-\mathrm{i} \diamond}\left\{\Pi^{H}\right\}\left\{\Delta^{<,>}\right\}-\mathrm{e}^{-\mathrm{i} \diamond}\left\{\Pi^{<,>}\right\}\left\{\Delta^{H}\right\}} \\
=\frac{1}{2} \mathrm{e}^{-\mathrm{i} \diamond}\left(\left\{\Pi^{>}\right\}\left\{\Delta^{<}\right\}-\left\{\Pi^{<}\right\}\left\{\Delta^{>}\right\}\right)
\end{gathered}
$$

and the equations for the retarded and advanced propagators

$$
\left[k^{2}+\mathrm{i} k^{0} \partial_{t}-\frac{1}{4} \partial_{t}^{2}-M^{2}\right] \Delta^{R, A}-\mathrm{e}^{-\mathrm{i} \diamond}\left\{\Pi^{H}\right\}\left\{\Delta^{R, A}\right\} \pm \mathrm{e}^{-\mathrm{i} \diamond}\left\{\mathrm{i} \Pi^{\mathcal{A}}\right\}\left\{\Delta^{R, A}\right\}=1 .
$$

In these equations, $\Delta=\Delta(k, t)$ and $\Pi=\Pi(k, t)$, and for the particular fields, we substitute $\Delta \rightarrow \Delta_{\chi, \varphi}, \Pi \rightarrow \Pi_{\chi, \varphi}$ and $M \rightarrow M_{\chi, \varphi}$. The derivation of these equations is discussed in detail in Refs. [48, 50 52]. As a quick reference, we list in Appendix A the definitions of the various Green functions and self energies as well as of the $\diamond$ operator, that appears in Wigner space. In order to keep the equations compact, we often suppress the momentum and time arguments of the Wigner functions in the following

Next, we keep only the zeroth order contributions in the operator $\diamond$, which we justify in more detail below. By taking the anti-hermitian and hermitian parts, it is useful to split the Kadanoff-Baym equations into the constraint,

$$
2\left[k^{2}-\frac{1}{4} \partial_{t}^{2}\right] \Delta^{<,>}-\left\{M^{2}+\Pi^{H}, \Delta^{<,>}\right\}-\left\{\Pi^{<,>}, \Delta^{H}\right\}=-\frac{1}{2}\left[\mathrm{i} \Pi^{>}, \mathrm{i} \Delta^{<}\right]+\frac{1}{2}\left[\mathrm{i} \Pi^{<}, \mathrm{i} \Delta^{>}\right],
$$

and the kinetic equations

$$
2 \mathrm{i} k^{0} \partial_{t} \Delta^{<,>}-\left[M^{2}, \Delta^{<,>}\right]=-\frac{1}{2}\left(\left\{\mathrm{i} \Pi^{>}, \mathrm{i} \Delta^{<}\right\}-\left\{\mathrm{i} \Pi^{<}, \mathrm{i} \Delta^{>}\right\}\right) .
$$

Of course, for the single-flavoured field $\varphi$, the commutator involving $M_{\varphi}^{2}$ vanishes.

The self-energies for $\chi$ are given by

$$
\mathrm{i}_{\chi_{i j}}^{a b}(p)=\int \frac{d^{4} k}{(2 \pi)^{4}}\left[g_{i} g_{j}^{*} \mathrm{i} \Delta_{\varphi}^{a b}(k) \mathrm{i} \Delta_{\varphi}^{a b}(p-k)+g_{i}^{*} g_{j} \mathrm{i} \Delta_{\varphi}^{b a}(-k) \mathrm{i} \Delta_{\varphi}^{b a}(k-p)\right]
$$

and for $\varphi$ by

$$
\mathrm{i}_{\varphi}^{a b}(p)=\sum_{i j} g_{i} g_{j}^{*} \int \frac{d^{4} k}{(2 \pi)^{4}} \mathrm{i} \Delta_{\chi_{i j}}^{a b}(k) \mathrm{i} \Delta_{\varphi}^{a b}(p-k)
$$

We absorb $\Pi^{H}$ into a redefinition of $M$ and can therefore effectively drop all terms involving it henceforth. 
Integration of the kinetic equations (5) for $\varphi$ yields the evolution of the charge asymmetry

$$
\begin{aligned}
& \int \frac{d p^{0}}{2 \pi} \mathrm{i} p^{0} \partial_{t} \mathrm{i} \Delta_{\varphi}^{<,>}=S_{\varphi}+W_{\varphi} \\
= & -\frac{1}{2} \int \frac{d p^{0}}{2 \pi}\left(\mathrm{i} \Pi_{\varphi}^{>}(p) \mathrm{i} \Delta_{\varphi}^{<}(p)-\mathrm{i}_{\varphi}^{<}(p) \mathrm{i} \Delta_{\varphi}^{>}(p)\right) \\
= & -\frac{1}{2} g_{i} g_{j}^{*} \int \frac{d p^{0}}{2 \pi} \int \frac{d^{4} k}{(2 \pi)^{4}}\left(\mathrm{i} \Delta_{\chi_{i j}}^{>}(k) \mathrm{i} \Delta_{\varphi}^{>}(p-k) \mathrm{i} \Delta_{\varphi}^{<}(p)-\mathrm{i} \Delta_{\chi_{i j}}^{<}(k) \mathrm{i} \Delta_{\varphi}^{<}(p-k) \mathrm{i} \Delta_{\varphi}^{>}(p)\right) .
\end{aligned}
$$

In this form, the equation encompasses both, the washout $W_{\varphi}$ of the charge of $\varphi$ as well as the source term for the asymmetry $S_{\varphi}$. When we assume that the charge density of $\varphi$ is small compared to the number density of its quasi-particles, a very useful simplification arises ( $c f$. Ref [33]). The washout term $W_{\varphi}$ can be approximated by including only the leading order effects, that do not involve $C P$-violation. For the $C P$-violating source $S_{\varphi}$, we may approximate the field $\varphi$ as following an equilibrium distribution. Decomposing

$$
\mathrm{i} \delta \Delta_{\chi_{i j}}=\mathrm{i} \Delta_{\chi_{i j}}^{<,>}-\mathrm{i} \Delta_{\chi_{i j}}^{<,>\text {eq }}
$$

and using KMS relations (124), we then find for the $C P$-violating source term

$$
S_{\varphi}=-g_{i} g_{j}^{*} \int \frac{d^{4} q}{(2 \pi)^{4}} \mathrm{i} \delta \Delta_{\chi_{i j}}(q) \hat{\Pi}_{\chi}^{\mathcal{A}}(q) .
$$

where we define

$$
\hat{\Pi}_{\chi}^{\mathcal{A}}=\Pi_{\chi_{i j}}^{\mathcal{A}} /\left(g_{i}^{*} g_{j}+g_{i} g_{j}^{*}\right)
$$

The spectral self-energy $\Pi_{\chi}^{\mathcal{A}}$ is a pivotal ingredient to calculations of the charge asymmetry within the CTP approach. In Appendix $\mathrm{B}$, the concrete expression for its equilibrium form is presented. Note that $\Pi_{\chi_{i i}}^{\mathcal{A}}(k) / k^{0}$ is the decay rate of $\chi_{i}$.

It remains to determine $i \delta \Delta_{\chi_{i j}}(q)$, the deviation of the field $\chi$ from equilibrium. From the constraint equations, we infer that the mass-diagonal components can be written as

$$
\mathrm{i} \delta \Delta_{\chi_{i i}}(p)=2 \pi \delta\left(p^{2}-M_{\chi_{i i}}^{2}\right)\left(\vartheta\left(p^{0}\right) \delta f_{\chi_{i i}}(\mathbf{p})+\vartheta\left(-p^{0}\right) \delta \bar{f}_{\chi_{i i}}(\mathbf{p})\right) .
$$

The functions $\delta f_{\chi}$ and $\delta \bar{f}_{\chi}$ describe the deviation of the quasi-particle distributions of the fields $\chi$ from their equilibrium Bose-Einstein form. Just as the Wigner functions, they are time-dependent, but we always suppress the time argument. For the diagonal components, the neutrality of the fields $\chi$, Eq. (105), implies that

$$
\delta f_{\chi_{i i}}(\mathbf{p})=\delta \bar{f}_{\chi_{i i}}(\mathbf{p}) .
$$

Notice that the terms within the constraint equations that involve $\Delta_{\chi}^{H}$ only contribute to the solution for the equilibrium Green function, but not to i $\delta \Delta_{\chi}$. For a single scalar flavour, this matter is explained in detail within Ref. [50]. 
It is useful to evaluate the kinetic and the constraint equations in a distributional sense, by integrating over some interval of $p^{0}$. For this purpose, we extract the distribution functions from the propagator. The neutrality condition (13) must be generalised for the off-diagonal components. In order to keep the notation compact, we therefore define

$$
\delta f_{\chi}(p)=\left\{\begin{array}{l}
\delta f_{\chi}(\mathbf{p}) \text { for } p^{0}>0 \\
\delta \bar{f}_{\chi}(\mathbf{p}) \text { for } p^{0}<0
\end{array},\right.
$$

i.e. $\delta f_{\chi}(p)$ depends on $p^{0}$ only through $\operatorname{sign}\left(p^{0}\right)$. We emphasise that this is just a convenient definition, because the values of $\delta f_{\chi}(p)$ for far off-shell momenta $p$ are irrelevant within our present approximations. We then extract these distribution functions through

$$
\delta f_{\chi}\left(p^{\prime 0}, \mathbf{p}\right)=\int_{\mathcal{I}_{ \pm}} \frac{d p^{0}}{2 \pi} 2 p^{0} \operatorname{sign}\left(p^{0}\right) \mathrm{i} \delta \Delta_{\chi}(p) \quad \forall p^{\prime 0} \mid \operatorname{sign}\left(p^{\prime 0}\right)= \pm .
$$

Here, $\mathcal{I}_{ \pm}$is a small interval around the positive (negative) quasi-particle pole. Note that the neutrality condition for scalar fields (105) and hermiticity (106) imply that

$$
\delta f_{\chi_{i j}}\left(p^{0}, \mathbf{p}\right)=\delta f_{\chi_{j i}}\left(-p^{0}, \mathbf{p}\right)=\delta f_{\chi_{i j}}^{*}\left(-p^{0}, \mathbf{p}\right) .
$$

Integrating the kinetic equations (5) according to Eq. (15), we obtain

$$
2 \mathrm{i} k^{0} \partial_{t} \delta f_{\chi}(k)-\left[M_{\chi}^{2}, \delta f_{\chi}(k)\right]=-\mathrm{i}\left\{\Pi^{\mathcal{A}}(k), \delta f_{\chi}(k)\right\} .
$$

These equations should be solved by setting $k^{0}$ to its value at the quasi-particle pole. The solution is $\delta f_{\chi}(\mathbf{k})$ for $k^{0}>0$ and $\delta \bar{f}_{\chi}(\mathbf{k})$ for $k^{0}<0$. It can then be completed according to Eq. (14), keeping in mind that $\delta f_{\chi}(k)$ is only physically meaningful for momenta close to the quasi-particle poles.

The off-diagonal components of i $\Delta_{\chi}$ can be determined based on two separate approximations, depending on the parametric regime. In order to define these regimes, we introduce

$$
\Gamma_{\mathrm{D}}(p) \approx \max _{i j}\left|\Pi_{\chi_{i j}}^{\mathcal{A}}(p) / p^{0}\right|
$$

as a measure of the width of the singlet $\chi$ or, equivalently, its decay rate. Besides, we define

$$
\begin{aligned}
\Delta M & =\left|M_{\chi_{11}}-M_{\chi_{22}}\right|, \\
\bar{M} & =\frac{M_{\chi_{11}}+M_{\chi_{22}}}{2} .
\end{aligned}
$$

The two parametric regimes are given by $\Delta M \gg \Gamma_{\mathrm{D}}$ and by $\Delta M \ll \bar{M}$. When $\chi$ is weakly coupled, as it is a typical requirement in scenarios for baryogenesis from out-of 
equilibrium decays, we have $\Gamma_{\mathrm{D}} \ll \bar{M}$, such that there is an overlap of both regimes when $\Gamma_{\mathrm{D}} \ll \Delta M \ll \bar{M}$. In that situation, both approximations should lead to the same predictions for the asymmetry.

The particular approximation strategies are now as follows:

- When $\Delta M \gg \Gamma_{\mathrm{D}}$, we can calculate the off-diagonal components of $\mathrm{i} \delta \Delta_{\chi}$ as higherorder corrections to the diagonal ones. For simplicity, we assume that only $\chi_{i}$ deviates from equilibrium. The general case follows from the superposition of the procedure that we describe here. In this approximation, the off-diagonal components share the diagonal mass-shells, i.e. the poles of these quasi-particles are given by

$$
\begin{array}{r}
\mathrm{i} \delta \Delta_{\chi_{i j}}(p)=2 \pi \delta\left(p^{2}-M_{\chi_{i i}}^{2}\right) \delta f_{\chi_{i j}}(p), \quad \mathrm{i} \delta \Delta_{\chi_{j i}}(p)=2 \pi \delta\left(p^{2}-M_{\chi_{i i}}^{2}\right) \delta f_{\chi_{j i}}(p) \\
\text { for } \Gamma_{\mathrm{D}} \ll \Delta M .
\end{array}
$$

As we show in Section 2.2, using either the kinetic or the constraint equations, it indeed follows that the off-diagonal components of the propagator are suppressed by factors of order $\Gamma_{\mathrm{D}} / \Delta M$ compared to the diagonal ones. These off-diagonal components then enter the source term $S_{\varphi}$ as given in Eq. (10), while higher order corrections to this are again suppressed by factors $\sim \Gamma_{\mathrm{D}} / \Delta M$. In principle, the same approximation strategy is employed in Ref. [30].

- When $\Delta M \ll \bar{M}$, we have no procedure yet in order to determine the precise location of the quasi-particle poles, but know that these are located at $p^{2}-\bar{M}^{2}$, up to a relative error of order $\Delta M / \bar{M}$. We can therefore approximate the off-diagonal out-of-equilibrium Wightman function as

$$
\mathrm{i} \delta \Delta_{\chi_{i j}}(p)=2 \pi \delta\left(p^{2}-\bar{M}^{2}\right) \delta f_{\chi_{i j}}(p) \quad \text { for } \Delta M \ll \bar{M} .
$$

When using this in order to solve the kinetic equation (17) as described above, we expect a relative error of order $\Delta M / \bar{M}$. Note that this approximation strategy is similar to the one that is employed in Refs. [53, 54].

In both cases, the result for i $\Delta_{\chi_{i j}}$ can then be substituted into the source term (10).

It is now the point to come back to the truncation of higher orders of the $\diamond$ operator, a procedure known as the gradient expansion. It is justified, provided we may neglect contributions to the finite width of the propagators, which are of relevance when the reaction $\chi \leftrightarrow 2 \varphi$ is kinematically forbidden [50]. Furthermore, contributions to the gradient expansion from fast flavour oscillations, which are systematically treated in Refs. 55 62, must not give sizable corrections to the phenomenological results. In the resonant regime, such corrections are suppressed by the small oscillation frequency and therefore effectively by factors of order $\Delta M / \bar{M}$, while in the non-resonant regime, the suppression results from the averaging of the oscillatory contribution to the source for the asymmetry, as we discuss below. Besides these considerations, the higher order terms 
in the gradient expansion yield contributions of order $H / T \sim T / m_{\mathrm{Pl}}$, where $H$ is the Hubble expansion rate, $m_{\mathrm{Pl}}$ the Planck mass and $T$ the temperature of the Universe, which sets the typical energy of a quasi-particle. For temperatures sufficiently below the Planck scale, corrections of this type should therefore be negligible.

\subsection{The Regime $\Delta M \gg \Gamma_{\mathrm{D}}$}

In this regime, when using the ansatz (20), we find the following approximate solution to the kinetic equations (17):

$$
\begin{aligned}
\delta f_{\chi_{i i}}(k) & =\delta f_{\chi_{i i}}^{0}(k) \mathrm{e}^{-\frac{\Pi_{\chi i i}^{\mathcal{A}}(k)}{k^{0}} t} \\
\delta f_{\chi_{i j}}(k) & =\mathrm{i} \frac{\Pi_{\chi_{i j}}^{\mathcal{A}}(k)}{M_{\chi_{i i}}^{2}-M_{\chi_{j j}}^{2}} \delta f_{\chi_{i i}}(k)=-\delta f_{\chi_{j i}}(k) .
\end{aligned}
$$

We assume here that only $\chi_{i}$ is excited by deviating from its equilibrium distribution, i.e. $\delta f_{\chi_{i i}}^{0}=\left.\delta f_{\chi_{i i}}\right|_{t=0},\left.\delta f_{\chi_{j j}}\right|_{t=0}=0$ and we set $k^{0}= \pm \sqrt{\mathbf{k}^{2}+M_{\chi_{i i}}^{2}}$. The case with an excited $\chi_{j}$ can be written down in analogous manner, taking account of the different underlying mass shell. As anticipated, the off-diagonal components are suppressed by a factor of order $\Gamma_{\mathrm{D}} / \Delta M$.

When substituting $\delta f_{\chi_{i j}}$ into the Wightman function according to Eq. (20) and eventually into the $C P$-violating source term (10) for $\varphi$, we recover the known answer for $\Delta M \gg \Gamma_{\mathrm{D}}$ and in finite density background:

$$
\begin{aligned}
S_{\varphi} & =-\mathrm{i} \int \frac{d^{4} q}{(2 \pi)^{4}} 2 \pi \delta\left(q^{2}-M_{\chi_{i i}}^{2}\right)\left(g_{i} g_{j}^{*}-g_{i}^{*} g_{j}\right) \frac{g_{i} g_{j}^{*}+g_{i}^{*} g_{j}}{M_{\chi_{i i}}^{2}-M_{\chi_{j j}}^{2}}\left(\hat{\Pi}_{\chi}^{\mathcal{A}}(q)\right)^{2} \delta f_{\chi_{i i}}(q) \\
& =-2 \mathrm{i}\left(g_{i}^{2} g_{j}^{* 2}-g_{i}^{* 2} g_{j}^{2}\right) \int \frac{d^{3} q}{(2 \pi)^{3} 2 \sqrt{\mathbf{q}^{2}+M_{\chi_{i i}}^{2}}} \frac{\left(\hat{\Pi}_{\chi}^{\mathcal{A}}(q)\right)^{2}}{M_{\chi_{i i}}^{2}-M_{\chi_{j j}}^{2}} \delta f_{\chi_{i i}}(\mathbf{q}),
\end{aligned}
$$

cf. Ref. [30].

Taking account of the time derivative (17) and of the anticommutator terms that involve the off-diagonal components of $f_{\chi}$, we obtain the leading finite-width corrections that appear within the denominator:

$$
\delta f_{\chi_{i j}}(k)=\mathrm{i} \frac{\Pi_{\chi_{i j}}^{\mathcal{A}}(k)}{M_{\chi_{i i}}^{2}-M_{\chi_{j j}}^{2}-\mathrm{i} \Pi_{\chi_{i i}}^{\mathcal{A}}+\mathrm{i} \Pi_{\chi_{j j}}^{\mathcal{A}}} \delta f_{\chi_{i i}}(k)=-\delta f_{\chi_{j i}}(k)
$$

Notice however, that this result still relies on the assumption $\Delta M \gg \Gamma_{\mathrm{D}}$.

The same result can be obtained when using the constraint equations (4). For given $\mathrm{i} \delta \Delta_{i i}$ (and assuming that $\mathrm{i} \delta \Delta_{i j}=0$ for $i \neq j$ at zeroth order in $\Gamma_{\mathrm{D}} / \Delta M$ ), the off-diagonal components follow from

$$
\left[2 k^{2}-\left(M_{\chi_{i i}}^{2}+M_{\chi_{j j}}^{2}\right)\right] \mathrm{i} \delta \Delta_{i j}=\mathrm{i} \Pi_{\chi_{i j}}^{\mathcal{A}} \mathrm{i} \delta \Delta_{\chi_{i i}}+\left(\mathrm{i} \Pi_{\chi_{i i}}^{\mathcal{A}}-\mathrm{i} \Pi_{\chi_{j j}}^{\mathcal{A}}\right) \mathrm{i} \delta \Delta_{i j}
$$


The crucial term on the right hand side arises from the commutator of the spectral self-energy and the distribution function and is neglected in earlier literature. Notice that the induced off-diagonal correlation is not oscillating. This is in contrast to earlier approximations, where the right hand side of the constraint equations is neglected [5563]. In conjunction with Eqs. (12) and (20), in particular with the on-shell condition $k^{2}=M_{\chi_{i i}}^{2}$, we find consistency with the solution (22b).

\subsection{The Regime $\Delta M \ll \bar{M}$ and Damped $2 \times 2$ Flavour Oscilla- tions in a Constant Background}

In this regime, the calculation should account for the potential impact of flavour oscillations. Scalar and fermionic fields share the same basic aspects of flavour dynamics. For the purpose of generalisation, we therefore recast Eq. (17) as

$$
\partial_{t} \delta f+\frac{\mathrm{i}}{2}[\Omega, \delta f]=-\frac{1}{2}\{\Gamma, \delta f\}
$$

where

$$
\Omega=M_{\chi}^{2} / k^{0}, \quad \Gamma=\Pi_{\chi}^{\mathcal{A}} / k^{0}
$$

and $\delta f=\delta f_{\chi}$. Of course, we can identify this with the evolution equation for a density matrix in the Hamiltonian formalism [19, 20, 23 26].

While this is not the case in the Early Universe, it is nonetheless instructive to consider the situation when $\Omega$ and $\Gamma$ are time-independent. The solution to equation (26) is then given by

$$
\delta f(t)=\mathrm{e}^{-\frac{i}{2} \Omega t-\frac{1}{2} \Gamma t} \delta f(t=0) \mathrm{e}^{\frac{i}{2} \Omega t-\frac{1}{2} \Gamma t} .
$$

Since $M_{\chi}$ is diagonal, we have chosen to work in a flavour basis where $\Omega$ is diagonal as well. In this basis, $\Gamma$ is non-diagonal in general, which is crucial in order to obtain $C P$-violating effects. Notice that $\Omega$ and $\Gamma$ are both hermitian. Let the matrix

$$
\Xi=\Omega-\mathrm{i} \Gamma
$$

be diagonalised by the transformation

$$
\Xi_{\mathrm{D}}=U \Xi U^{-1}
$$

We choose the parametrisation (see e.g. Ref. [67])

$$
U=\left(\begin{array}{cc}
c & t_{1} c \\
-t_{2} c & c
\end{array}\right)
$$


where

$$
\begin{aligned}
\Delta & =\frac{1}{2}\left(\Xi_{11}-\Xi_{22}\right), & D & =\operatorname{sign}(\operatorname{Re}[\Delta]) \sqrt{\Delta^{2}+\Xi_{12} \Xi_{21}}, \\
t_{1} & =\frac{\Xi_{12}}{\Delta+D}, & t_{2} & =\frac{\Xi_{21}}{\Delta+D}, \\
c & =\frac{1}{\sqrt{1+t_{1} t_{2}}}, & \delta & =\frac{\Xi_{12} \Xi_{21}}{\Delta+D}, \\
\Xi_{\mathrm{D} 11} & =\Xi_{11}+\delta, & \Xi_{\mathrm{D} 22} & =\Xi_{22}-\delta .
\end{aligned}
$$

The matrix

$$
\Xi^{\mathrm{c}}=\Omega+\mathrm{i} \Gamma
$$

is diagonalised by

$$
\Xi_{\mathrm{D}}^{\mathrm{c}}=V \Xi^{\mathrm{c}} V^{-1},
$$

where $V$ can be constructed in the same way as $U$. Notice that $\Xi_{\mathrm{D}}^{c}=\Xi_{\mathrm{D}}^{*}, U^{\dagger}=V^{-1}$ and $\operatorname{det} U=1$.

We can thus express

$$
\delta f(t)=U^{-1} \mathrm{e}^{-\mathrm{i} \Xi_{\mathrm{D}} t} U \delta f(0) V^{-1} \mathrm{e}^{\mathrm{i} \Xi_{\mathrm{D}}^{\mathrm{c}} t} V=U^{-1} \mathrm{e}^{-\mathrm{i} \Xi_{\mathrm{D}} t} U \delta f(0) U^{\dagger} \mathrm{e}^{\mathrm{i} \Xi_{\mathrm{D}}^{\mathrm{c}} t} U^{\dagger^{-1}} .
$$

Note that $\delta f(t)$ is hermitian by construction, provided $\delta f(0)$ is. From this formula, we can infer the distribution functions of $\chi$ and their correlations as a function of time in a straightforward way.

For this purpose, appropriate boundary conditions for $\delta f$ need to be specified. In order to choose these, it is useful to first consider the equilibrium propagators. We express these various propagators in terms of their adjugate matrices

$$
\begin{aligned}
& \Delta_{\chi}^{R}=\frac{1}{k^{0^{2}}-\omega^{2}+\mathrm{i} \Pi_{\chi}^{\mathcal{A}}}=\frac{1}{\mathcal{D}} \operatorname{adj}\left(k^{0^{2}}-\omega^{2}+\mathrm{i} \Pi_{\chi}^{\mathcal{A}}\right), \\
& \Delta_{\chi}^{A}=\frac{1}{k^{0^{2}}-\omega^{2}-\mathrm{i} \Pi_{\chi}^{\mathcal{A}}}=\frac{1}{\mathcal{D}^{*}} \operatorname{adj}\left(k^{0^{2}}-\omega^{2}-\mathrm{i} \Pi_{\chi}^{\mathcal{A}}\right), \\
& \Delta_{\chi}^{\mathcal{A}}=\frac{\mathrm{i}}{2}\left(\Delta_{\chi}^{R}-\Delta_{\chi}^{A}\right)=\frac{\mathrm{i}}{2|\mathcal{D}|^{2}}\left[\operatorname{adj}\left(k^{0^{2}}-\omega^{2}\right)\left(\mathcal{D}-\mathcal{D}^{*}\right)+\mathrm{i} \operatorname{adj}\left(\Pi_{\chi}^{\mathcal{A}}\right)\left(\mathcal{D}+\mathcal{D}^{*}\right)\right], \\
& \Delta_{\chi}^{H}=\frac{1}{2}\left(\Delta_{\chi}^{R}+\Delta_{\chi}^{A}\right)=\frac{\mathrm{i}}{2|\mathcal{D}|^{2}}\left[\operatorname{adj}\left(k^{0^{2}}-\omega^{2}\right)\left(\mathcal{D}+\mathcal{D}^{*}\right)+\mathrm{i} \operatorname{adj}\left(\Pi_{\chi}^{\mathcal{A}}\right)\left(\mathcal{D}-\mathcal{D}^{*}\right)\right] \text {, }
\end{aligned}
$$

where

$$
\mathcal{D}=\operatorname{det}\left[k^{0^{2}}-\omega^{2}+i \Pi_{\chi}^{\mathcal{A}}\right]
$$

and $\omega^{2}=\mathbf{k}^{2}+M_{\chi}^{2}$. These solutions can be obtained from the equations for the retarded and advanced propagators (3). Besides, the equilibrium Wigner functions i $\Delta_{\chi}^{<e q}(k)=$ 
$2 \Delta_{\chi}^{\mathcal{A}}(k) /\left(\exp \left(\beta k^{0}\right)-1\right)$ and i $\Delta_{\chi}^{>\mathrm{eq}}(k)=2 \Delta_{\chi}^{\mathcal{A}}(k)\left[1+1 /\left(\exp \left(\beta k^{0}\right)-1\right)\right]$ can be consistently obtained as solutions to the constraint equations (44) as well, $c f$. Ref. [50] for a detailed discussion.

As we assume a mass-diagonal basis, where $\omega^{2}$ is diagonal, it becomes clear that in the hierarchical limit, this is indeed the appropriate basis for defining the density of quasi-particles. In particular, notice that for $\Delta M_{\chi_{i j}} \gg \Gamma$, it follows

$$
\mathrm{i} \Delta_{\chi_{i i}}^{\mathcal{A}} \approx \frac{\Pi_{\chi_{i i}}^{\mathcal{A}}}{\left(k^{0^{2}}-\omega_{i i}^{2}\right)^{2}+\Pi_{\chi_{i i}}^{\mathcal{A}}}
$$

while

$$
\mathrm{i} \Delta_{\chi_{i j}}^{\mathcal{A}} \approx \Pi_{\chi_{i j}}^{\mathcal{A}}\left(\frac{1}{\left(k^{0^{2}}-\omega_{i i}^{2}\right)^{2}+\Pi_{\chi_{i i}}^{\mathcal{A}}{ }^{2}} \frac{k^{0^{2}}-\omega_{i i}^{2}}{k^{0^{2}}-\omega_{j j}^{2}}+\frac{1}{\left(k^{0^{2}}-\omega_{j j}^{2}\right)^{2}+\Pi_{\chi_{j j}}^{\mathcal{A}}{ }^{2}} \frac{k^{0^{2}}-\omega_{j j}^{2}}{k^{0^{2}}-\omega_{i i}^{2}}\right),
$$

where $\omega_{i i}^{2}=\mathbf{k}^{2}+M_{\chi_{i i}}^{2}$. Hence, the diagonal contributions are of the Breit-Wigner type, as required by the spectral sum rule

$$
\int \frac{d k^{0}}{2 \pi} k^{0} \Delta_{i j}^{\mathcal{A}}=\delta_{i j}
$$

while the off-diagonal entries are representations of the principal value that cannot be associated with particle number.

Therefore, while it is tempting to choose for Eq. (35) initial conditions where $U \delta f(0) V^{-1}$ is a diagonal matrix, because then $\delta f(t)$ has no oscillatory contributions, we should instead choose $\delta f(0)$ diagonal in order to describe a state that initially exhibits no mixing of the mass eigenstates. For illustrative purposes, we therefore consider a situation where $\chi_{1}$ is out-of-equilibrium, while $\chi_{2}$ is in equilibrium, i.e.

$$
\delta f(0)=\left(\begin{array}{cc}
\delta f_{0} & 0 \\
0 & 0
\end{array}\right) .
$$

Eq. (35) then yields

$$
\begin{aligned}
& \delta f_{11}(t)=\delta f_{0} \frac{\left(\left(\Delta^{*}+D^{*}\right)^{2} \mathrm{e}^{\frac{\mathrm{i}}{2} \Xi_{D 11}^{\mathrm{c}} t}-\left|\Gamma_{12}\right|^{2} \mathrm{e}^{\frac{\mathrm{i}}{2} \Xi_{D 22}^{\mathrm{c}} t}\right)\left((\Delta+D)^{2} \mathrm{e}^{-\frac{\mathrm{i}}{2} \Xi_{D 11} t}-\left|\Gamma_{12}\right|^{2} \mathrm{e}^{-\frac{\mathrm{i}}{2} \Xi_{D 22} t}\right)}{\left.\left.\left|(\Delta+D)^{2}-\right| \Gamma_{12}\right|^{2}\right|^{2}}, \\
& \delta f_{12}(t)=\delta f_{0} \frac{\mathrm{i} \Gamma_{12}\left(\Delta^{*}+D^{*}\right)\left(\mathrm{e}^{\frac{\mathrm{i} \Xi^{\mathrm{c}}}{D 11} t}-\mathrm{e}^{\frac{\mathrm{i}}{2} \Xi_{D 22}^{\mathrm{c}} t}\right)\left((\Delta+D)^{2} \mathrm{e}^{-\frac{\mathrm{i}}{2} \Xi_{D 11} t}-\left|\Gamma_{12}\right|^{2} \mathrm{e}^{-\frac{i}{2} \Xi_{D 22} t}\right)}{\left.\left.\left|(\Delta+D)^{2}-\right| \Gamma_{12}\right|^{2}\right|^{2}}, \\
& \delta f_{21}(t)=\delta f_{12}^{*}(t), \\
& \delta f_{22}(t)=\delta f_{0} \frac{\left|\Gamma_{12}\right|^{2}|\Delta+D|^{2}\left(\mathrm{e}^{\frac{i}{2} \Xi_{D 11}^{c} t}-\mathrm{e}^{\frac{i}{2} \Xi_{D 22}^{c} t}\right)\left(\mathrm{e}^{-\frac{i}{2} \Xi_{D 11} t}-\mathrm{e}^{-\frac{i}{2} \Xi_{D 22} t}\right)}{\left.\left.\left|(\Delta+D)^{2}-\right| \Gamma_{12}\right|^{2}\right|^{2}} .
\end{aligned}
$$


In the $C P$-violating source term for the scalar model (10), this enters when using Eq. (21).

It is interesting to study the behaviour of the solutions (28) and (42) in the degenerate limit, where $M_{\chi_{11}}=M_{\chi_{22}}$. Because $\Pi_{\chi}^{\mathcal{A}}$ is real symmetric, $\Gamma_{12}$ is real. We can then see from Eq. (10), that the $C P$-violating source $S_{\varphi}$ is proportional to the imaginary part of $\delta f_{12}$. In Eq. (28), $\Omega$ commutes with all terms for $\Delta M=0$, since it is proportional to the identity matrix. Therefore, no contribution to $S_{\varphi}$ arises in that situation. From Eq. (42b), we arrive at the same conclusion, because both $\Delta$ and $D$ are purely imaginary for $\Delta M=0$ and $\operatorname{Re}\left[\Xi_{\mathrm{D} 11}\right]=\operatorname{Re}\left[\Xi_{\mathrm{D} 22}\right]$. Therefore, $\delta f_{12}(t)$ turns out to be real, which implies that $S_{\varphi}=0$.

We should also comment on the case where $(\Delta+D)^{2}-\left|\Gamma_{12}\right|^{2}=0$. While from Eq. (28), it is immediately clear that the result for $\delta f(t)$ must be non-singular also in this limit, we need to explicitly convince ourselves of the cancellation of singularities in Eqs. (42). From Eqs. (321), we find that $\Delta^{2}=\Xi_{12} \Xi_{21}=\left|\Gamma_{12}\right|^{2}, D=0$ and $\Xi_{\mathrm{D} 11}=\Xi_{\mathrm{D} 22}=\Xi_{11}-\sqrt{-\Xi_{12} \Xi_{21}}$ in that situation. Substitution into Eqs. (42) then explicitly reveals the cancellation. In conclusion, we find $S_{\varphi}=0$ for $\Delta M=0$, even when the fields $\chi_{1,2}$ are distinguishable through their interactions $g_{1,2}$ with the field $\varphi$. Of course, one can alternatively arrive at the same conclusion through a field redefinition through a unitary transformation of $\chi$, that may remove the phases from the couplings $g$ but leaves $M_{\chi}$ invariant for $\Delta M=0$.

Finally, for $\Delta M \gg \Gamma_{\mathrm{D}}$, we can cross check the solutions (42b) by neglecting the oscillatory contributions based on the assumption that these average out in the final asymmetry, as we discuss in Section 4. In that case, we find

$$
\delta f_{12}=\frac{\mathrm{i} \Pi_{12}^{\mathcal{A}}}{M_{\chi_{11}}^{2}-M_{\chi_{22}}^{2}} \delta f_{0} \mathrm{e}^{-\frac{\Pi_{11}^{\mathcal{A}}}{k^{0}} t},
$$

which is in agreement with the result (22), where the approximation of non-degeneracy has been applied at an earlier stage of the calculation.

\section{Fermions}

\subsection{CTP Approach to Leptogenesis}

For our treatment of Leptogenesis, we follow Ref. [33], where the CTP approach in the non-resonant regime is formulated. A lot of that material applies to the resonant regime as well, which is the main objective of the present work. Besides, we employ the results of Ref. [33] as a benchmark in order to test the results that are derived here. Because they are applicable arbitrarily close to the limit of mass-degenerate singlet neutrinos, the present results are a generalisation of those presented in Ref. [33], and we show that both agree sufficiently far away from the mass degeneracy. Notice that we do not reiterate the calculation of the asymmetry from the vertex (penguin) correction in the present paper.

The Lagrangian that gives rise to the standard scenario of Leptogenesis in the unflavoured regime is

$$
\mathcal{L}=\frac{1}{2} \bar{\psi}_{N i}\left(\mathrm{i} \not \partial-M_{i j}\right) \psi_{N j}+\bar{\psi}_{\ell} \mathrm{i} \not \psi_{\ell}+\left(\partial^{\mu} \phi^{\dagger}\right)\left(\partial_{\mu} \phi\right)-Y_{i}^{*} \bar{\psi}_{\ell} \phi^{\dagger} P_{\mathrm{R}} \psi_{N i}-Y_{i} \bar{\psi}_{N i} P_{\mathrm{L}} \phi \psi_{\ell} .
$$


Here, $N_{i}$ are the singlet right-handed Majorana neutrinos, that correspond to the fields $\chi_{i}$ in the scalar model. Their masses are given by the mass matrix $M$, for which we choose a diagonal basis, unless explicitly stated otherwise. Again, we take $i=1$, 2 , for simplicity, while a generalisation to more flavours of $N$ is straightforward. The Standard Model Higgs doublet field is $\phi$, and $\ell$ is a linear combination of the three left-handed lepton doublets of the Standard Model. $\psi_{\ell}$ and $\psi_{N}$ are the spinors associated with $\ell$ and $N$. Note that the diagonal form of the Yukawa couplings $Y$, that is assumed in the Lagrangian (44), can be achieved by a unitary transformation of the left-handed lepton flavours. The $\mathrm{SU}(2)_{\mathrm{L}}$ indices are contracted in a gauge-invariant way, i.e. $\phi \psi_{\ell}=$ $-\phi_{A} \epsilon_{A B} \psi_{\ell B}$ and $\bar{\psi}_{\ell} \phi^{\dagger}=\left(\bar{\psi}_{\ell}\right)_{A} \epsilon_{A B}\left(\phi^{\dagger}\right)_{B}$, where $\epsilon_{A B}$ is the antisymmetric $2 \times 2$ tensor with $\epsilon_{12}=1$. The four-component spinor $\psi_{N i}$ observes the Majorana constraint (108). The chiral projectors are $P_{\mathrm{L}, \mathrm{R}}=\left(1 \mp \gamma_{5}\right) / 2$.

In the Lagrangian of the form (44), $M$ may be a general complex symmetric matrix. Through field redefinitions of $N$, it is possible to bring $M$ to a real diagonal form, along with an according reparametrisation of $Y_{i}$. Yet, once a basis is chosen where $M$ is real and diagonal in the vacuum, thermal corrections may lead to a real symmetric effective mass that adds to $M$. The off-diagonal components can then be be removed through an addtional orthogonal transformation, but in general not in a temperature-independent way. Yet, choosing a basis where $M$ is real and symmetric, has the advantage that the $C P$-violating phases are isolated within $Y_{i}$. Moreover, the separation of the KadanoffBaym equations into kinetic and constraint equations by taking the hermitian and antihermitian parts is facilitated when $M$ is purely hermitian. For these reasons, we continue the discussion under the assumption that $M$ is real and symmetric.

The propagators for the left-handed lepton and the Higgs field are listed in Appendix C. At zeroth order in perturbation theory, the diagonal components of the singlet neutrino propagators are parametrised in terms of the distribution functions $f_{N}$ as

$$
\begin{aligned}
& \mathrm{i} S_{N i i}^{<}(p)=-2 \pi \delta\left(p^{2}-M_{i i}^{2}\right)\left(\not p+M_{i i}\right)\left[\vartheta\left(p_{0}\right) f_{N i i}(\mathbf{p})-\vartheta\left(-p_{0}\right)\left(1-f_{N i i}(-\mathbf{p})\right)\right], \\
& \mathrm{i} S_{N i i}^{>}(p)=-2 \pi \delta\left(p^{2}-M_{i i}^{2}\right)\left(\not p+M_{i i}\right)\left[-\vartheta\left(p_{0}\right)\left(1-f_{N i i}(\mathbf{p})\right)+\vartheta\left(-p_{0}\right) f_{N i i}(-\mathbf{p})\right] \\
& \mathrm{i} S_{N i i}^{T}(p)=\frac{\mathrm{i}\left(\not p+M_{i i}\right)}{p^{2}-M_{i i}^{2}+\mathrm{i} \varepsilon}-2 \pi \delta\left(p^{2}-M_{i i}^{2}\right)\left(\not p+M_{i i}\right)\left[\vartheta\left(p_{0}\right) f_{N i i}(\mathbf{p})+\vartheta\left(-p_{0}\right) f_{N i i}(-\mathbf{p})\right] \\
& \mathrm{i} S_{N i i}^{\bar{T}}(p)=-\frac{\mathrm{i}\left(\not p+M_{i i}\right)}{p^{2}-M_{i i}^{2}-\mathrm{i} \varepsilon}-2 \pi \delta\left(p^{2}-M_{i i}^{2}\right)\left(\not p+M_{i i}\right)\left[\vartheta\left(p_{0}\right) f_{N i i}(\mathbf{p})+\vartheta\left(-p_{0}\right) f_{N i i}(-\mathbf{p})\right] .
\end{aligned}
$$

In general, $f_{N}$ decomposes into equilibrium and out-of-equilibrium contributions as $f_{N}=f_{N}^{\text {eq }}+\delta f_{N}$, where $f_{N}^{\text {eq }}$ is a Fermi-Dirac distribution. For the equilibrium contributions to i $S_{N}$, we could then derive finite-width corrections, $c f$. Eqs. (36) and Ref. [50] for the scalar case. As these corrections are not relevant for the present analysis, we do not include them here. Notice that the propagators (45) observe the Majorana constraint (109). 
We aim for approximate solutions to the the Kadanoff-Baym equations for the singlet neutrinos, which are

$$
\begin{aligned}
& \left(\not k+\frac{\mathrm{i}}{2} \not \partial-M_{i k}\right) S_{N_{k j}}^{<,>}-\mathrm{e}^{-\mathrm{i} \diamond}\left\{\not_{N_{i k}}^{H}\right\}\left\{S_{N_{k j}}^{<,>}\right\}-\mathrm{e}^{-\mathrm{i} \diamond}\left\{\not{H}_{N_{i k}}^{<,>}\right\}\left\{S_{N_{k j}}^{H}\right\} \\
= & \frac{1}{2} \mathrm{e}^{-\mathrm{i} \diamond}\left(\left\{\mathbb{Z}_{N_{i k}}^{>}\right\}\left\{S_{N_{k j}}^{<}\right\}-\left\{\mathbb{Z}_{N_{i k}}^{<}\right\}\left\{S_{N_{k j}}^{>}\right\}\right),
\end{aligned}
$$

with the self energy

$$
\begin{aligned}
\mathrm{i}_{N i j}^{a b}(k)= & g_{w} \int \frac{d^{4} k^{\prime}}{(2 \pi)^{4}} \frac{d^{4} k^{\prime \prime}}{(2 \pi)^{4}}(2 \pi)^{4} \delta^{(4)}\left(k-k^{\prime}-k^{\prime \prime}\right) \\
& \times\left\{Y_{i} Y_{j}^{*} P_{\mathrm{L}} \mathrm{i} S_{\ell}^{a b}\left(k^{\prime}\right) P_{\mathrm{R}} \mathrm{i} \Delta_{\phi}^{a b}\left(k^{\prime \prime}\right)+Y_{i}^{*} Y_{j} C\left[P_{\mathrm{L}} \mathrm{i} S_{\ell}^{b a}\left(-k^{\prime}\right) P_{\mathrm{R}}\right]^{t} C^{\dagger} \mathrm{i} \Delta_{\phi}^{b a}\left(-k^{\prime \prime}\right)\right\} .
\end{aligned}
$$

Through the factor $g_{w}=2$, we take account of the $\mathrm{SU}(2)_{\mathrm{L}}$ multiplicity. Since Ref. [33] omits a discussion of how this self-energy can be obtained from the two-particle-irreducible (2PI) effective action approach, that is often used in non-equilibrium field theory, we briefly present this connection in Appendix D ( $c f$. also Ref. [68]). In analogy with the discussion of the scalar case, the hermitian part of the self-energy $\not_{N}^{H}$ can eventually be absorbed in a redefinition of $M^{2}$, that appears below in the effective equation (95) for the evolution of the distribution function for the $N_{i}$. Technically, this can be performed by generalising the methods for Weyl fermions in Ref. [34] to the case of massive Majorana fermions. The contribution that results from $\mathbb{K}_{N}^{H}$ is real symmetric, but temperature dependent. In general, it is therefore not possible to diagonalise the effective mass in a temperature-independent way. The temperature-dependent term is given by Eq. (96) below.

When replacing the definitions (18) and (19) by

$$
\Gamma_{\mathrm{D}}(p) \approx \max _{i j}\left|\operatorname{tr}\left[\not p \mathbb{K}_{N_{i j}^{\mathcal{A}}}^{\mathcal{A}}(p)\right] / p^{0}\right|
$$

and

$$
\begin{aligned}
\Delta M & =\left|M_{11}-M_{22}\right|, \\
\bar{M} & =\frac{M_{11}+M_{22}}{2},
\end{aligned}
$$

we can identify the same basic approximation strategies for the calculation of the lepton asymmetry from mixing Majorana neutrinos with the calculation of the asymmetry from mixing neutral scalars, that is discussed in Section 2. In particular, it is useful to make use of the approximation $\Gamma_{\mathrm{D}} \ll \Delta M$, where the crucial off-diagonal components of the singlet neutrino Green functions can be obtained as small perturbations to the diagonal ones, and of the approximation $\Delta M \ll \bar{M}$, where the off-diagonal Green functions can be obtained from the kinetic equations. Fortunately, since $\bar{M} \gg \Gamma_{\mathrm{D}}$, due to the smallness of 
the couplings $Y$, the parametric regions, where these conditions hold, overlap, such that phenomenologically reliable predictions are available throughout the parameter space.

We should now specify the pivotal quantity $\mathbb{k}_{N}^{\mathcal{A}}$. Since for the massless lepton, i $S_{\ell}$ is proportional to $\gamma$-matrices, the same holds true for the self-energy. In particular, we may write

$$
\gtrless_{N i j}^{\mathcal{A}}(k)=\gamma^{\mu} g_{w}\left(Y_{i}^{*} Y_{j} \hat{\Sigma}_{N \mathrm{~L} \mu}^{\mathcal{A}} P_{\mathrm{L}}+Y_{i} Y_{j}^{*} \hat{\Sigma}_{N \mathrm{R} \mu}^{\mathcal{A}} P_{\mathrm{R}}\right)
$$

The expressions for $\hat{\Sigma}_{N \mathrm{~L}, \mathrm{R}}^{\mathcal{A} \mu}$ with lepton and Higgs distributions in kinetic equilibrium, i.e. for Fermi-Dirac and Bose-Einstein distributions with chemical potentials $\mu_{\ell}$ and $\mu_{\phi}$, are given by Eqs. (114). When $\Delta M \gg \Gamma_{\mathrm{D}}$ is not fulfilled, the off-diagonal correlations of the right-handed neutrinos can effectively retain lepton number over an amount of time that can be relevant for Leptogenesis. In order to accurately treat the washout of the asymmetry, it is therefore important to account for the chemical potentials $\mu_{\ell}$ and $\mu_{\phi}$ when solving for the Kadanoff-Baym equations for the singlet neutrino propagator (46). The extreme case is a setup considered in Ref. [69], where the right-handed neutrinos and their interactions approximately conserve lepton number. On the other hand, when $\Delta M \gg \Gamma_{\mathrm{D}}$ holds, it is a good approximation to neglect the lepton and Higgs chemical potential for the purpose of calculating $\mathfrak{Z}_{N}^{\mathcal{A}}$, such that we can set

$$
\left.\Sigma_{N}^{\mathcal{A} \mu} \equiv \Sigma_{N_{\mathrm{L}}}^{\mathcal{A} \mu}\right|_{\mu_{\ell}=\mu_{\phi}=0}=\left.\Sigma_{N_{\mathrm{R}}}^{\mathcal{A} \mu}\right|_{\mu_{\ell}=\mu_{\phi}=0}
$$

and 2

$$
\gtrless_{N i j}^{\mathcal{A}}(k)=\gamma_{\mu} \hat{\Sigma}_{N}^{\mathcal{A} \mu} g_{w}\left(Y_{i} Y_{j}^{*} P_{\mathrm{R}}+Y_{i}^{*} Y_{j} P_{\mathrm{L}}\right)
$$

Note that $\frac{1}{4} \operatorname{tr}\left[\not k \psi_{N i i}^{\mathcal{A}}\right] / k^{0}$ is the decay rate of $N_{i}$.

The one-loop lepton self energy is

$$
\mathrm{i} \sum_{\ell}^{\mathrm{wf}<,>}(k)=\int \frac{d^{4} k^{\prime}}{(2 \pi)^{4}} \frac{d^{4} k^{\prime \prime}}{(2 \pi)^{4}}(2 \pi)^{4} \delta^{4}\left(k-k^{\prime}-k^{\prime \prime}\right) Y_{i}^{*} Y_{j} P_{\mathrm{R}} \mathrm{i} S_{N i j}^{\mathrm{wf}<,>}\left(k^{\prime}\right) P_{\mathrm{L}} \mathrm{i} \Delta_{\phi}^{>,<}\left(-k^{\prime \prime}\right),
$$

which drives the evolution of the lepton asymmetry according to

$$
\frac{d}{d t}\left(n_{\ell}-\bar{n}_{\ell}\right)=S+W=\int \frac{d^{4} k}{(2 \pi)^{4}} \operatorname{tr}\left[\mathrm{i} Z_{\ell}^{>}(k) P_{\mathrm{L}} \mathrm{i} S_{\ell}^{<}(k)-\mathrm{i} \not_{\ell}^{<}(k) P_{\mathrm{L}} \mathrm{i} S_{\ell}^{>}(k)\right],
$$

where $n_{\ell}\left(\bar{n}_{\ell}\right)$ is the number density of (anti-) leptons. We decompose this expression into the source term $S$ and the washout term $W$. The source is extracted, when substituting equilibrium propagators for $\mathrm{i} S_{\ell}$ and i $\Delta_{\phi}$. This approximation is valid, provided the lepton charge density is small compared to the number density. In particular, we do not need to account for the lepton and Higgs chemical potentials when calculating the source term.

\footnotetext{
${ }^{2}$ This definition is related to the similar quantity in Ref. [33] through $\hat{\Sigma}_{N}^{\mathcal{A} \mu}=\frac{1}{2 g_{w}} \Sigma_{N}^{\mu}$.
} 
The KMS relations then imply, that the source is proportional to the deviation of the singlet neutrino from equilibrium,

$$
\begin{aligned}
S= & Y_{i}^{*} Y_{j} \int \frac{d^{4} k}{(2 \pi)^{4}} \int \frac{d^{4} k^{\prime}}{(2 \pi)^{4}} \int \frac{d^{4} k^{\prime \prime}}{(2 \pi)^{4}}(2 \pi)^{4} \delta^{4}\left(k-k^{\prime}-k^{\prime \prime}\right) \\
& \times \operatorname{tr}\left[P_{\mathrm{R}} \mathrm{i} \delta S_{N_{i j}}\left(k^{\prime}\right) P_{\mathrm{L}}\left(\mathrm{i} \Delta_{\phi}^{<}\left(-k^{\prime \prime}\right) \mathrm{i} S_{\ell}^{<}(k)-\mathrm{i} \Delta_{\phi}^{>}\left(-k^{\prime \prime}\right) \mathrm{i} S_{\ell}^{>}(k)\right)\right] \\
= & -Y_{i}^{*} Y_{j} \int \frac{d^{4} k^{\prime}}{(2 \pi)^{4}} \operatorname{tr}\left[P_{\mathrm{R}} \mathrm{i} \delta S_{N_{i j}}\left(k^{\prime}\right) 2 P_{\mathrm{L}} \hat{\dot{\psi}}_{N}^{\mathcal{A}}\left(k^{\prime}\right)\right],
\end{aligned}
$$

with equilibrium distributions and vanishing chemical potentials for $\ell$ and $\phi$, such that $\hat{\Sigma}_{N}^{\mathcal{A}}$ is given by Eq. (52). A complete network of equations that determine the evolution of the lepton charge in the expanding Universe is presented in Section 5 .

\subsection{Helicity Block-Diagonal Decomposition of the Singlet Neu- trino Propagator}

In order to explicitly demonstrate the consistency of the constraint and the kinetic equations when $\Delta M \gg \Gamma_{\mathrm{D}}$ and in order to pave the ground for the calculation of the asymmetry when $\Delta M \ll \bar{M}$, we decompose the fermionic Green functions into various Dirac matrix components. In particular, for a spatially homogeneous and isotropic problem, it is useful to notice that the helicity operator $\hat{h}=\hat{k}^{i} \gamma^{0} \gamma^{i} \gamma^{5}$ commutes with the various Dirac matrices that appear in the Kadanoff-Baym equations (46), such that helicity is a good quantum number. This suggests the decomposition [51, 70, 71]

$$
\mathrm{i} \delta S_{N}=\sum_{h= \pm} \mathrm{i} \delta S_{N h}, \quad-\mathrm{i} \gamma^{0} \delta S_{N h}=\frac{1}{4}\left(\mathbb{1}+h \hat{k}^{i} \sigma^{i}\right) \otimes \rho^{a} g_{a h}
$$

where $h= \pm$ denotes helicity and $\sigma^{i}, \rho^{i}$ are Pauli matrices. Note that the functions $g_{a h}$ are understood to be hermitian matrices in flavour space. It may be useful to compare with Eq. (129), in order to acquire a quick understanding of the functions $g_{a h}$.

Multiplication of the Kadanoff-Baym equations (46) by $\left\{\mathbb{1},-h \gamma^{i} \gamma^{5},-\mathrm{i} h \gamma^{i},-\gamma^{5}\right\}$, taking the Dirac trace and truncating the diamond operators yields the equations

$$
\left[\mathcal{F}_{h}-\mathbf{Y}^{*} \mathbf{Y}^{t} \mathcal{C}_{h \mathrm{~L}}-\mathbf{Y} \mathbf{Y}^{\dagger} \mathcal{C}_{h \mathrm{R}}\right]\left(\begin{array}{c}
g_{0 h} \\
g_{1 h} \\
g_{2 h} \\
g_{3 h}
\end{array}\right)=0,
$$

where $\mathbf{Y}^{t}=\left(Y_{1}, Y_{2}\right)$,

$$
\mathcal{F}_{h}=\left(\begin{array}{cccc}
-\mathrm{ik}^{0}+\frac{1}{2} \partial_{t} & \mathrm{i} M & 0 & \mathrm{i} h|\mathbf{k}| \\
\mathrm{i} M & -\mathrm{ik}^{0}+\frac{1}{2} \partial_{t} & h|\mathbf{k}| & 0 \\
0 & -h|\mathbf{k}| & -\mathrm{ik}^{0}+\frac{1}{2} \partial_{t} & M \\
\mathrm{i} h|\mathbf{k}| & 0 & -M & -\mathrm{ik}^{0}+\frac{1}{2} \partial_{t}
\end{array}\right),
$$


and

$$
\begin{aligned}
& \mathcal{C}_{h \mathrm{~L}, \mathrm{R}}=\frac{g_{w}}{2} \times
\end{aligned}
$$

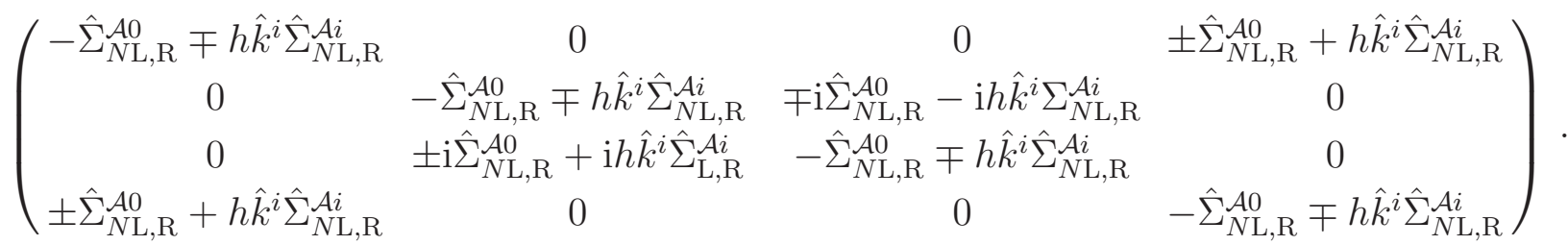

The kinetic equations correspond to the hermitian part of Eqs. (57), which is

$$
\begin{aligned}
\dot{g}_{0 h}+\mathrm{i}\left[M, g_{1 h}\right] & =\sum_{ \pm}-\frac{g_{w}}{2}\left(\hat{\Sigma}_{N \mathrm{~L}, \mathrm{R}}^{\mathcal{A} 0} \pm h \hat{k}^{i} \hat{\Sigma}_{N \mathrm{~L}, \mathrm{R}}^{\mathcal{A} i}\right)\left\{\Upsilon_{\mathrm{L}, \mathrm{R}}, g_{0 h} \mp g_{3 h}\right\}, \\
\dot{g}_{1 h}+2 h|\mathbf{k}| g_{2 h}+\mathrm{i}\left[M, g_{0 h}\right] & =\sum_{ \pm} \frac{g_{w}}{2}\left(\hat{\Sigma}_{N \mathrm{~L}, \mathrm{R}}^{\mathcal{A} 0} \pm h \hat{k}^{i} \hat{\Sigma}_{N \mathrm{~L}, \mathrm{R}}^{\mathcal{A} i}\right)\left(-\left\{\Upsilon_{\mathrm{L}, \mathrm{R}}, g_{1 h}\right\} \mp \mathrm{i}\left[\Upsilon_{\mathrm{L}, \mathrm{R}}, g_{2 h}\right]\right), \\
\dot{g}_{2 h}-2 h|\mathbf{k}| g_{1 h}+\left\{M, g_{3 h}\right\} & =\sum_{ \pm} \frac{g_{w}}{2}\left(\hat{\Sigma}_{N \mathrm{~L}, \mathrm{R}}^{\mathcal{A} 0} \pm h \hat{k}^{i} \hat{\Sigma}_{N \mathrm{~L}, \mathrm{R}}^{\mathcal{A} i}\right)\left( \pm \mathrm{i}\left[\Upsilon_{\mathrm{L}, \mathrm{R}}, g_{1 h}\right]-\left\{\Upsilon_{\mathrm{L}, \mathrm{R}}, g_{2 h}\right\}\right), \\
\dot{g}_{3 h}-\left\{M, g_{2 h}\right\} & =\sum_{ \pm}-\frac{g_{w}}{2}\left(\hat{\Sigma}_{N \mathrm{~L}, \mathrm{R}}^{\mathcal{A} 0} \pm h \hat{k}^{i} \hat{\Sigma}_{N \mathrm{~L}, \mathrm{R}}^{\mathcal{A} i}\right)\left\{\Upsilon_{\mathrm{L}, \mathrm{R}}, g_{3 h} \mp g_{0 h}\right\},
\end{aligned}
$$

where we define the shorthand notations

$$
\Upsilon_{\mathrm{L}}=\mathbf{Y}^{*} \mathbf{Y}^{t}, \quad \Upsilon_{\mathrm{R}}=\mathbf{Y} \mathbf{Y}^{\dagger}
$$

The constraint equations are the anti-hermitian part of Eqs. (57) and read

$$
\begin{aligned}
-2 \mathrm{i} k^{0} g_{0 h}+2 \mathrm{i} h|\mathbf{k}| g_{3 h}+\mathrm{i}\left\{M, g_{1 h}\right\} & =\sum_{ \pm}-\frac{g_{w}}{2}\left(\hat{\Sigma}_{N \mathrm{~L}, \mathrm{R}}^{\mathcal{A} 0} \pm h \hat{k}^{i} \hat{\Sigma}_{N \mathrm{~L}, \mathrm{R}}^{\mathcal{A} i}\right)\left[\Upsilon_{\mathrm{L}, \mathrm{R}}, g_{0 h} \mp g_{3 h}\right] \\
-2 \mathrm{i} k^{0} g_{1 h}+\mathrm{i}\left\{M, g_{0 h}\right\} & =\sum_{ \pm} \frac{g_{w}}{2}\left(\hat{\Sigma}_{N \mathrm{~L}, \mathrm{R}}^{\mathcal{A} 0} \pm h \hat{k}^{i} \hat{\Sigma}_{N \mathrm{~L}, \mathrm{R}}^{\mathcal{A} i}\right) \\
& \times\left(-\left[\Upsilon_{\mathrm{L}, \mathrm{R}}, g_{1 h}\right] \mp \mathrm{i}\left\{\Upsilon_{\mathrm{L}, \mathrm{R}}, g_{2 h}\right\}\right) \\
-2 \mathrm{i} k^{0} g_{2 h}+\left[M, g_{3 h}\right] & =\sum_{ \pm} \frac{g_{w}}{2}\left(\hat{\Sigma}_{N \mathrm{~L}, \mathrm{R}}^{\mathcal{A} 0} \pm h \hat{k}^{i} \hat{\Sigma}_{N \mathrm{~L}, \mathrm{R}}^{\mathcal{A} i}\right) \\
& \times\left( \pm \mathrm{i}\left\{\Upsilon_{\mathrm{L}, \mathrm{R}}, g_{1 h}\right\}-\left[\Upsilon_{\mathrm{L}, \mathrm{R}}, g_{2 h}\right]\right) \\
-2 \mathrm{i} k^{0} g_{3 h}+2 \mathrm{i} h|\mathbf{k}| g_{0 h}-\left[M, g_{2 h}\right] & =\sum_{ \pm}-\frac{g_{w}}{2}\left(\hat{\Sigma}_{N \mathrm{~L}, \mathrm{R}}^{\mathcal{A} 0} \pm h \hat{k}^{i} \hat{\Sigma}_{N \mathrm{~L}, \mathrm{R}}^{\mathcal{A} i}\right)\left[\Upsilon_{\mathrm{L}, \mathrm{R}}, g_{3 h} \mp g_{0 h}\right]
\end{aligned}
$$




\subsection{Derivation of the Asymmetry for $\Delta M \gg \Gamma_{\mathrm{D}}$}

In the regime $\Delta M \gg \Gamma_{\mathrm{D}}$, we calculate the off-diagonal components of $\mathrm{i} \delta S_{N i j}$ as small perturbations for a given diagonal out-of-equilibrium distribution. We verify that constraint and kinetic equations give consistent answers. This is in analogy with the procedure that is presented in Section 2.2 for the scalar case, and the error is controlled by the expansion parameter $\Gamma_{\mathrm{D}} / \Delta M$.

We first derive the source of the lepton asymmetry from the constraint equations (62). Assuming a mass-diagonal basis and neglecting the collision term, we obtain from the diagonal components of Eqs. (62) the relations

$$
g_{1 h i i}(k)=\frac{M_{i i}}{k^{0}} g_{0 h i i}(k), \quad g_{2 h i i}(k)=0, \quad g_{3 h i i}(k)=\frac{h|\mathbf{k}|}{k^{0}} g_{0 h i i}(k) .
$$

For these diagonal components, we assume helicity-independence, i.e. $g_{0+i i}=g_{0-i i}$. This follows when assuming that there is no initial helicity asymmetry within the singlet neutrinos and no initial charge asymmetry within the lepton and Higgs sector (and hence, no $C P$-asymmetry). Because the tree-level decays and inverse decays then produce no helicity asymmetry, also the non-equilibrium distributions for the $N_{i}$ are helicity symmetric at leading order. A helicity asymmetry in $N_{i}$ is only incurred through $C P$ violating loop effects and the back-reaction of the produced lepton asymmetry, which results in sub-leading contributions to the non-equilibrium distributions for the $N_{i}$. Note that symmetric initial conditons are naturally established within the parametric range of strong washout. Through Eqs. (45), we may relate $g_{0 h i i}$ to the distribution functions as

$$
\operatorname{sign}\left(k^{0}\right) 2 \sqrt{\mathbf{k}^{2}+M_{i i}^{2}} \delta f_{N_{i i}}(\mathbf{k}) 2 \pi \delta\left(k^{2}-M_{i i}^{2}\right)=g_{0 h i i}(k) .
$$

From the expression for the source term (55), we see that we need to determine the offdiagonal components (that is $i \neq j$ ) of $P_{\mathrm{R}} \mathrm{i} \delta S_{N i j} P_{\mathrm{L}}$. Therefore, we employ the diagonal solutions to derive the off-diagonal contributions as perturbations of order $\Gamma_{\mathrm{D}} / \Delta M$. Because $\Delta M \gg \Gamma_{\mathrm{D}}$, lepton number is rapidly violated once the inverse decay into a righthanded neutrino has occurred. We can therefore neglect the lepton and Higgs chemical potentials when calculating the singlet propagator and use the approximation (51). The 
constraint equations for the off-diagonal components $(i \neq j)$ are then given by

$$
\begin{aligned}
&-2 \mathrm{i} k^{0} g_{0 h i j}^{\mathrm{L}, \mathrm{R}}+2 \mathrm{i} h|\mathbf{k}| g_{3 h i j}^{\mathrm{L}, \mathrm{R}}+\mathrm{i}\left(M_{i i}+M_{j j}\right) g_{1 h i j}^{\mathrm{L}, \mathrm{R}}=\frac{1}{2}\left(\Sigma_{N i j}^{\mathcal{A} 0} \pm h \hat{k}^{i} \Sigma_{N i j}^{\mathcal{A} i}\right)\left(g_{0 h i i} \mp g_{3 h i i}\right) \\
&-2 \mathrm{i} k^{0} g_{1 h i j}^{\mathrm{L}, \mathrm{R}}+\mathrm{i}\left(M_{i i}+M_{j j}\right) g_{0 h i j}^{\mathrm{L}, \mathrm{R}}=\frac{1}{2}\left(\Sigma_{N i j}^{\mathcal{A} 0} \pm h \hat{k}^{i} \Sigma_{N i j}^{\mathcal{A} i}\right)\left(g_{1 h i i} \mp \mathrm{i} g_{2 h i i}\right) \\
&-2 \mathrm{i} k^{0} g_{2 h i j}^{\mathrm{L}, \mathrm{R}}+\left(M_{i i}-M_{j j}\right) g_{3 h i j}^{\mathrm{L}, \mathrm{R}}= \pm \frac{1}{2} \mathrm{i}\left(\Sigma_{N i j}^{\mathcal{A} 0} \pm h \hat{k}^{i} \Sigma_{N i j}^{\mathcal{A} i}\right)\left(g_{1 h i i} \mp \mathrm{i} g_{2 h i i}\right) \\
&-2 \mathrm{i} k^{0} g_{3 h i j}^{\mathrm{L}, \mathrm{R}}+2 \mathrm{i} h|\mathbf{k}| g_{0 h i j}^{\mathrm{L}, \mathrm{R}}-\left(M_{i i}-M_{j j}\right) g_{2 h i j}^{\mathrm{L}, \mathrm{R}}=\mp \frac{1}{2}\left(\Sigma_{N i j}^{\mathcal{A} 0} \pm h \hat{k}^{i} \Sigma_{N i j}^{\mathcal{A} i}\right)\left(g_{0 h i i} \mp g_{3 h i i}\right)
\end{aligned}
$$

where $g_{0,3 h i j}^{\mathrm{L}}=Y_{i}^{*} Y_{j} \hat{g}_{0,3 h i j}^{\mathrm{L}}, g_{0,3 h i j}^{\mathrm{R}}=Y_{j}^{*} Y_{i} \hat{g}_{0,3 h i j}^{\mathrm{R}}$ and $g_{0,3 h i j}=g_{0,3 h i j}^{\mathrm{L}}+g_{0,3 h i j}^{\mathrm{R}}$. Because of hermiticity, $g_{0,3 h j i}^{\mathrm{L}, \mathrm{R}}=\left(g_{0,3 h i j}^{\mathrm{L}, \mathrm{R}}\right)^{*}$. It is assumed here, that only $g_{h i i} \neq 0$, while $g_{h j j}=0$. The general solution can be constructed by superposition.

Given the diagonal solutions (ㅎ3), we can now straightforwardly determine the $g_{a h i j}$ for $i \neq j$ as solutions to the system of linear equations (65). In particular, the flavour off-diagonal vector- and pseudovector-densities are

$$
\begin{aligned}
\hat{g}_{0 h 12}^{\mathrm{L}, \mathrm{R}} & =\frac{M_{11}\left(M_{11}+M_{22}\right) \mp 2 h|\mathbf{k}| k^{0}+2 \mathbf{k}^{2}}{k^{0}\left(M_{11}^{2}-M_{22}^{2}\right)} \mathrm{i} \frac{g_{w}}{2}\left(\hat{\Sigma}_{N}^{\mathcal{A} 0} \pm h \hat{k}^{i} \hat{\Sigma}_{N}^{\mathcal{A} i}\right) g_{0 h 11}, \\
\hat{g}_{3 h 12}^{\mathrm{L}, \mathrm{R}} & =\frac{M_{11}\left(M_{11}-M_{22}\right) \mp 2 h|\mathbf{k}| k^{0}+2 \mathbf{k}^{2}}{k^{0}\left(M_{11}^{2}-M_{22}^{2}\right)} \mathrm{i} \frac{g_{w}}{2}\left(\mp \hat{\Sigma}_{N}^{\mathcal{A} 0}-h \hat{k}^{i} \hat{\Sigma}_{N}^{\mathcal{A} i}\right) g_{0 h 11} .
\end{aligned}
$$

We have now assumed that only $N_{1}$ deviates from equilibrium. As stated above, the general case, where $N_{2}$ deviates as well, can simply be obtained by superposition.

Note that these sub-leading off-diagonal solutions do not obey the leading-order symmetry property, according to which $g_{0 h}$ is helicity-even whereas $g_{3 h}$ is helicity-odd. For the $C P$-violating source, above results combine to a compact form that observes the symmetries of the problem:

$$
\begin{aligned}
Y_{i}^{*} Y_{j} P_{\mathrm{R}} \mathrm{i} \delta S_{N_{i j}}(k) P_{\mathrm{L}} & =-Y_{i}^{*} Y_{j} \frac{1}{4} \sum_{h= \pm}\left[P_{\mathrm{R}} \gamma^{0} P_{\mathrm{L}}-h P_{\mathrm{R}} \hat{k}^{i} \gamma^{i} P_{\mathrm{L}}\right]\left(g_{0 h i j}+g_{3 h i j}\right) \\
& =-\left(Y_{1}^{* 2} Y_{2}^{2}-Y_{1}^{2} Y_{2}^{* 2}\right) \frac{\mathrm{i}}{k^{0}} \frac{M_{11} M_{22}}{M_{11}^{2}-M_{22}^{2}} P_{\mathrm{R}} \gamma^{\mu} P_{\mathrm{L}} \frac{g_{w}}{2} \hat{\Sigma}_{N \mu}^{\mathcal{A}} g_{0 h 11} \\
& =-\left(Y_{1}^{* 2} Y_{2}^{2}-Y_{1}^{2} Y_{2}^{* 2}\right) \mathrm{i} \frac{M_{11} M_{22}}{M_{11}^{2}-M_{22}^{2}} \frac{g_{w}}{2} P_{\mathrm{R}} \hat{\psi}_{N}^{\mathcal{A}} P_{\mathrm{L}} 2 \delta f_{N 11}(\mathbf{k}) 2 \pi \delta\left(k^{2}-M_{1}^{2}\right)
\end{aligned}
$$

Since $N_{2}$ is assumed to be in equilibrium, $\delta f_{N 22}=0$. Notice that only contributions from $\hat{g}_{0 h 11}^{\mathrm{L}}$ enter here, while those from $\hat{g}_{0 h 11}^{\mathrm{R}}$ cancel since they are multiplied by the conjugate 
Yukawa couplings. Substituting Eq. (67) into Eq. (55), we obtain the known result for the $C P$-violating source of the lepton asymmetry [33],

$$
S=\int \frac{d^{3} k}{(2 \pi)^{3} 2 \sqrt{\mathbf{k}^{2}+M_{11}^{2}}} 8 \mathrm{i}\left[Y_{1}^{* 2} Y_{2}^{2}-Y_{1}^{2} Y_{2}^{* 2}\right] \frac{M_{11} M_{22}}{M_{11}^{2}-M_{22}^{2}} g_{w} \hat{\Sigma}_{N \mu}^{\mathcal{A}} \hat{\Sigma}_{N}^{\mathcal{A} \mu} \delta f_{N_{11}}(\mathbf{k}),
$$

which includes the finite-density corrections. We refer to this expression as the perturbative result for the source term, as it can be calculated as a perturbation to the diagonal singlet neutrino propagator or, alternatively, from a loop expansion of Feynman diagrams as in Ref. [33].

While above result is obtained from the constraint equations (62), we now check the consistency with the kinetic equations (60) when using the same approximations: When $\Delta M \gg \Gamma_{\mathrm{D}}$, the diagonal (11)-densities track the deviation of $N_{1}$ from equilibrium, which provides the thermodynamical breakdown of time-reversal invariance, which is necessary for baryogenesis. Compared to these diagonal components, the off-diagonal ones are suppressed by a factor of order $\Gamma_{\mathrm{D}} / \Delta M$. In the absence of flavour oscillations, the timederivatives of the off-diagonals are additionally suppressed by the same parameter, such that we may neglect them. With these approximations, the resulting set of equations obtained from the (12)-components of Eqs. (60) is

$$
\begin{aligned}
\mathrm{i}\left(M_{11}-M_{22}\right) g_{1 h 12}^{\mathrm{L}, \mathrm{R}} & =-\frac{1}{2}\left(\Sigma_{N 12}^{\mathcal{A} 0} \pm h \hat{k}^{i} \Sigma_{N 12}^{\mathcal{A} i}\right)\left(g_{0 h 11} \mp g_{3 h 11}\right), \\
2 h|\mathbf{k}| g_{2 h 12}^{\mathrm{L}, \mathrm{R}}+\mathrm{i}\left(M_{11}-M_{22}\right) g_{0 h 12}^{\mathrm{L}, \mathrm{R}} & =-\frac{1}{2}\left(\Sigma_{N 12}^{\mathcal{A} 0} \pm h \hat{k}^{i} \Sigma_{N 12}^{\mathcal{A} i}\right)\left(g_{1 h 11} \mp \mathrm{i} g_{2 h 11}\right), \\
-2 h|\mathbf{k}| g_{1 h 12}^{\mathrm{L}, \mathrm{R}}+\left(M_{11}+M_{22}\right) g_{3 h 12}^{\mathrm{L}, \mathrm{R}} & =\mp \frac{1}{2} \mathrm{i}\left(\Sigma_{N 12}^{\mathcal{A} 0} \pm h \hat{k}^{i} \Sigma_{N 12}^{\mathcal{A} i}\right)\left(g_{1 h 11} \mp \mathrm{i} g_{2 h 11}\right), \\
-\left(M_{11}+M_{22}\right) g_{2 h 12}^{\mathrm{L}, \mathrm{R}} & = \pm \frac{1}{2}\left(\Sigma_{N 12}^{\mathcal{A} 0} \pm h \hat{k}^{i} \Sigma_{N 12}^{\mathcal{A} i}\right)\left(g_{0 h 11} \mp g_{3 h 11}\right) .
\end{aligned}
$$

Indeed, from these equations, we can straightforwardly reproduce the result (66) .

\subsection{Asymmetry for $\Delta M \ll \bar{M}$}

In order to obtain a solution for the $C P$-violating source also in the regime where $\Delta M$ is of the same order as $\Gamma_{\mathrm{D}}$ or smaller, we could aim for a full solution of the kinetic equations (60). Within an intuitive picture of flavour oscillations, the meaning of Eqs. (60) is yet somewhat obscure because of the mixing of the $g_{a h}$, which is a result of the spinor structure. A diagonalisation of this spinor structure is not possible in general, because the dispersive contributions from $M$ and the absorptive ones from $\sharp_{N}^{\mathcal{A}}$ cannot be diagonalised simultaneously.

In the close-to-degenerate regime, where flavour oscillations are important, crucial simplifications can however be achieved. We do not need to specify the locations of the quasi-particle poles of the diagonal and off-diagonal components of the propagators exactly, but only make use of the information that these are located at $p^{2}=\bar{M}^{2}$, up to 
a relative error of order $\Delta M / \bar{M}$. We can then integrate the constraint and the kinetic equations (60) over an interval $\mathcal{I}$ of length of order $\Delta M$ over the region of the quasiparticle poles. As a result, we obtain again the kinetic equations (60) and the constraint equations (62) with the replacement $g_{a h i j} \rightarrow \delta f_{a h i j}$, where

$$
\int_{\mathcal{I}_{ \pm}} \frac{d p^{0}}{2 \pi} \operatorname{sign}\left(p^{0}\right) g_{a h i j}(p)= \begin{cases}\delta f_{a h i j}(\mathbf{p}) & \text { for }+ \\ \delta \bar{f}_{a h i j}(\mathbf{p}) & \text { for }-\end{cases}
$$

and $\mathcal{I}_{ \pm}$is again an interval around the positive or negative energy quasi-particle pole, respectively. The error results from the averaging over values of $p^{0}$ in the constraint equations and can be estimated to be of relative order $\Delta M / \bar{M}$. In order to keep the notation compact when we have to distinguish the cases of positive and negative frequencies, we adapt the pragmatic definition (14) to the fermionic case,

$$
\delta f_{a h i j}(p)=\left\{\begin{array}{l}
\delta f_{a h i j}(\mathbf{p}) \text { for } p^{0}>0 \\
\delta \bar{f}_{a h i j}(\mathbf{p}) \text { for } p^{0}<0
\end{array} .\right.
$$

When $\Gamma_{\mathrm{D}} \ll \bar{M}$ and $\Delta M \ll \bar{M}$, it follows from Eq. (60c) or from Eqs. (62) that

$$
\delta f_{1 h i j}(k)=\delta f_{3 h i j}(k) \frac{M_{i i}+M_{j j}}{2 h|\mathbf{k}|}
$$

and from Eq. (62b) that

$$
\delta f_{1 h i j}(k)=\delta f_{0 h i j}(k) \frac{M_{i i}+M_{j j}}{2 k^{0}}
$$

Using these approximate constraints, Eq. (60a) becomes

$$
\begin{aligned}
\delta \dot{f}_{0 h}+\frac{1}{2 k^{0}} \mathrm{i}\left[M^{2}, \delta f_{0 h}\right] & =-\sum_{ \pm} \frac{g_{w}}{2}\left(\hat{\Sigma}_{N \mathrm{~L}, \mathrm{R}}^{\mathcal{A} 0} \pm h \hat{k}^{i} \hat{\Sigma}_{N \mathrm{~L}, \mathrm{R}}^{\mathcal{A} i}\right)\left(1 \mp \frac{h|\mathbf{k}|}{k^{0}}\right)\left\{\Upsilon_{\mathrm{L}, \mathrm{R}}, \delta f_{0 h}\right\} \\
& =-g_{w}\left\{\operatorname{Re}\left[\mathbf{Y}^{*} \mathbf{Y}^{t}\right] \frac{k \cdot \hat{\Sigma}_{N}^{\mathcal{A}}}{k^{0}}-\mathrm{i} h \operatorname{Im}\left[\mathbf{Y}^{*} \mathbf{Y}^{t}\right] \frac{\tilde{k} \cdot \hat{\Sigma}_{N}^{\mathcal{A}}}{k^{0}}, \delta f_{0 h}\right\},
\end{aligned}
$$

where $\tilde{k} \equiv\left(|\mathbf{k}|, k^{0} \hat{k}\right)$ and in the last row we have neglected the lepton and Higgs chemical potentials according to Eq. (51). As discussed above, the latter approximation is not in general justified when $\Delta M \lesssim \Gamma_{\mathrm{D}}$. The off-diagonal components of the out-of-equilibrium Wightman function for the singlet neutrinos can then be approximated as

$$
g_{a h i j}(p)=2 \pi \delta\left(p^{2}-\bar{M}^{2}\right) 2 p^{0} \delta f_{a h i j}(p)
$$

which is accurate up to a relative error of order $\Delta M / \bar{M}$. In order to calculate the $C P$-asymmetric source, this should then be substituted in Eq. (56) and eventually in Eq. (155). 
We can easily perform the consistency check that Eq. (74) reproduces above results when $\Gamma_{\mathrm{D}} \ll \bar{M}$ and $\Delta M \ll \bar{M}$ but $\Delta M \gg \Gamma_{\mathrm{D}}$. Neglecting the lepton and Higgs chemical potentials according to Eq. (51), the off-diagonal terms that arise from the out-of-equilibrium singlet neutrino $N_{1}$ are

$$
\begin{aligned}
\delta f_{0 h 12}= & 2 \mathrm{i}\left[Y_{1}^{*} Y_{2} \frac{k^{0}-h|\mathbf{k}|}{M_{11}^{2}-M_{22}^{2}} \frac{g_{w}}{2}\left(\hat{\Sigma}_{12}^{\mathcal{A} 0}+h \hat{k}^{i} \hat{\Sigma}_{12}^{\mathcal{A}}\right)\right. \\
& \left.+Y_{1} Y_{2}^{*} \frac{k^{0}+h|\mathbf{k}|}{M_{11}^{2}-M_{22}^{2}} \frac{g_{w}}{2}\left(\hat{\Sigma}_{N}^{\mathcal{A} 0}-h \hat{k}^{i} \hat{\Sigma}_{N}^{\mathcal{A} i}\right)\right] \delta f_{0 h 11}, \\
\delta f_{3 h 12}= & 2 \mathrm{i}\left[Y_{1}^{*} Y_{2} \frac{\mathbf{k}^{2}-h|\mathbf{k}| k^{0}}{k^{0}\left(M_{11}^{2}-M_{22}^{2}\right)} \frac{g_{w}}{2}\left(-\hat{\Sigma}_{N}^{\mathcal{A} 0}-h \hat{k}^{i} \hat{\Sigma}_{N}^{\mathcal{A} i}\right)\right. \\
& \left.+Y_{1} Y_{2}^{*} \frac{\mathbf{k}^{2}+h|\mathbf{k}| k^{0}}{k^{0}\left(M_{11}^{2}-M_{22}^{2}\right)} \frac{g_{w}}{2}\left(\hat{\Sigma}_{N}^{\mathcal{A} 0}-h \hat{k}^{i} \hat{\Sigma}_{N}^{\mathcal{A} i}\right)\right] \delta f_{0 h 11},
\end{aligned}
$$

where we have neglected the time-derivatives on the left-hand side of Eq. (74). Therefore, these equations cannot take into account possible flavour oscillations for the $N_{i}$, which should however be irrelevant for the lepton asymmetry when $\Delta M \gg \Gamma_{\mathrm{D}}$, as we explain in Section 4. Note that these expressions can as well be obtained from Eqs. (66) when taking $M_{11} \rightarrow M_{22}$ and using the definition (70). As a further consistency check, we also note that in the regime where $\Delta M \gg \Gamma_{\mathrm{D}}$ but $\Delta M \ll \bar{M}$, we can exchange $M_{11} \leftrightarrow M_{22}$ within Eqs. (63) and $M_{11}+M_{22} \leftrightarrow 2 M_{11,22}$ in Eqs. (60). Within the result (66) for $g_{0 h 12}$ and $g_{3 h 12}$, this indeed only incurs a relative error of order $\Delta M / \bar{M}$, which is a small correction.

Now, when we relax the requirement that $\Delta M \gg \Gamma_{\mathrm{D}}$, Eqs. (74) can be straightforwardly solved numerically. Besides, it is also instructive to obtain analytical solutions in a situation without expansion of the Universe. These can be obtained when substituting

$$
\begin{aligned}
\Omega=\frac{M^{2}}{k^{0}} \quad \text { and } \quad \Gamma & =2 \sum_{ \pm} \frac{g_{w}}{2} \Upsilon_{\mathrm{L}, \mathrm{R}}\left(\hat{\Sigma}_{N \mathrm{~L}, \mathrm{R}}^{\mathcal{A} 0} \pm h \hat{k}^{i} \hat{\Sigma}_{N \mathrm{~L}, \mathrm{R}}^{\mathcal{A} i}\right)\left(1 \mp \frac{h|\mathbf{k}|}{k^{0}}\right) \\
& =2 g_{w}\left(\operatorname{Re}\left[\mathbf{Y}^{*} \mathbf{Y}^{t}\right] \frac{k \cdot \hat{\Sigma}_{N}^{\mathcal{A}}}{k^{0}}-\mathrm{i} h \operatorname{Im}\left[\mathbf{Y}^{*} \mathbf{Y}^{t}\right] \frac{\tilde{k} \cdot \hat{\Sigma}_{N}^{\mathcal{A}}}{k^{0}}\right)
\end{aligned}
$$

in Eq. (26). In the last row of Eq. (177) we have again neglected the lepton and Higgs chemical potentials according to Eq. (51). Before we discuss some features of these solutions in Section 4 , we note an additional consistency check. When neglecting oscillatory contributions, as it is appropriate for $\Gamma_{\mathrm{D}} \ll \Delta M$, we can immediately recover the perturbative solution (76a) from Eq. (42b). Eq. (76b) is then obtained through $g_{3 h}=\left(h|\mathbf{k}| / k^{0}\right) g_{0 h}$, which also holds for the flavour off-diagonals in the close-todegenerate regime, as we have demonstrated above in Eqs. (72)|73). When $\Delta M \gg \Gamma_{\mathrm{D}}$, we therefore consistently reproduce the result for the lepton number violating contribution to the off-diagonal singlet-neutrino Wightman function (67) and eventually the well-known result for the $C P$-violating source (68). 
We have therefore established that for $\Delta M \gg \Gamma_{\mathrm{D}}$, the kinetic equations (26) accurately reproduce the classic result for the resonantly enhanced lepton asymmetry [3, 6 10], that arises as the zero-temperature limit of Eq. (68). In order to achieve this agreement, the helicity structure exhibited in Eqs. (74) and (77) is crucial. For the lepton asymmetry from decaying Majorana neutrinos, to our knowledge, this is the first time an explicit connection between results from the $S$-matrix approach and from kinetic evolution equations is established.

\section{Illustrative Features of Analytical Solutions when Ignoring Washout and the Expanding Background}

We specialise here to the regime where the approximation $\Delta M \ll M$ is valid and first consider the scalar model. Therefore, we use the form (21) for the off-diagonal Wightman functions of $\chi$. The source term for the asymmetry (10) can then be expressed as

$$
S_{\varphi}=-g_{i} g_{j}^{*} \int \frac{d^{3} q}{(2 \pi)^{3} 2 \sqrt{\mathbf{q}^{2}+\bar{M}^{2}}} \sum_{q^{0}= \pm \omega(\mathbf{q})} \hat{\Pi}_{\chi}^{\mathcal{A}}(q) \delta f_{\chi_{i j}}(q) \text {. }
$$

When adding the positive and negative energy contributions, it is useful to notice the transformation properties of the quantities in Eqs. (32)

$$
\begin{gathered}
\Delta \underset{q^{0} \rightarrow-q^{0}}{\longrightarrow}-\Delta^{*}, \\
D \underset{q^{0} \rightarrow-q^{0}}{\longrightarrow}-D^{*}, \\
\Xi_{\mathrm{D} i i} \underset{q^{0} \rightarrow-q^{0}}{\longrightarrow}-\Xi_{\mathrm{D} i i}^{*} .
\end{gathered}
$$

As a consequence, $\delta f_{\chi_{i j}}\left(k^{0}, \mathbf{k}\right)=\delta f_{\chi_{j i}}\left(-k^{0}, \mathbf{k}\right)$, a relation that can also be derived from the neutrality condition (105) imposed on i $\Delta_{\chi}$. Use of the result (42) then yields

$$
\begin{aligned}
S_{\varphi} & =-\mathrm{i}\left(g_{1}^{2} g_{2}^{* 2}-g_{1}^{* 2} g_{2}^{2}\right) \int \frac{d^{3} q}{(2 \pi)^{3} 2 \sqrt{\mathbf{q}^{2}+\bar{M}^{2}}} \delta f_{0}(\mathbf{q}) \frac{\hat{\Pi}_{\chi}^{\mathcal{A}^{2}}}{\left|(\Delta+D)^{2}-\right| \Pi_{\chi_{12}}^{\mathcal{A}} /\left.\left.\omega(\mathbf{q})\right|^{2}\right|^{2}} \\
& \times\left[\left(\Delta^{*}+D^{*}\right)\left(\mathrm{e}^{\frac{\mathrm{i}}{2} \Xi_{\mathrm{D} 11}^{\mathrm{c}} t}-\mathrm{e}^{\frac{i}{2} \Xi_{\mathrm{D} 22}^{\mathrm{c}} t}\right)\left((\Delta+D)^{2} \mathrm{e}^{-\frac{\mathrm{i}}{2} \Xi_{\mathrm{D} 11} t}-\left|\Pi_{\chi_{12}}^{\mathcal{A}} / \omega(\mathbf{q})\right|^{2} \mathrm{e}^{-\frac{i}{2} \Xi_{\mathrm{D} 22} t}\right)+\text { c.c. }\right],
\end{aligned}
$$

where we have used that $\Pi_{\chi}^{\mathcal{A}}\left(k^{0}, \mathbf{k}\right)=-\Pi_{\chi}^{\mathcal{A}}\left(-k^{0}, \mathbf{k}\right), \omega(\mathbf{q})=\sqrt{\mathbf{q}^{2}+\bar{M}^{2}}$ and we evaluate $\Pi^{\mathcal{A}}=\Pi^{\mathcal{A}}(\omega(\mathbf{q}), \mathbf{q})$. The parameters $\Delta, D$ and $\Xi$ are given by Eqs. (27,32)

In order to assess the role of the oscillatory contributions within this source term, we 
integrate

$$
\begin{aligned}
\int_{0}^{\infty} d t S_{\varphi} & =-\mathrm{i}\left(g_{1}^{2} g_{2}^{* 2}-g_{1}^{* 2} g_{2}^{2}\right) \int \frac{d^{3} q}{(2 \pi)^{3} 2 \sqrt{\mathbf{q}^{2}+\bar{M}^{2}}} \delta f_{0}(\mathbf{q}) \frac{\hat{\Pi}_{\chi}^{\mathcal{A}^{2}}}{\left|(\Delta+D)^{2}-\right| \Pi_{\chi 12}^{\mathcal{A}} /\left.\left.\omega(\mathbf{q})\right|^{2}\right|^{2}} \\
& \times\left[|\Delta+D|^{2}(\Delta+D)\left(\frac{1}{\Gamma_{\mathrm{D} 11}}-\frac{1}{\frac{\mathrm{i}}{2}\left(\Omega_{\mathrm{D} 11}-\Omega_{\mathrm{D} 22}\right)-\frac{1}{2}\left(\Gamma_{\mathrm{D} 11}-\Gamma_{\mathrm{D} 22}\right)}\right)\right. \\
& \left.-\left(\Delta^{*}+D^{*}\right)\left|\frac{\Pi_{\chi 12}^{\mathcal{A}}}{\omega(\mathbf{q})}\right|^{2}\left(\frac{1}{\frac{\mathrm{i}}{2}\left(\Omega_{\mathrm{D} 11}-\Omega_{\mathrm{D} 22}\right)-\frac{1}{2}\left(\Gamma_{\mathrm{D} 11}-\Gamma_{\mathrm{D} 22}\right)}-\frac{1}{\Gamma_{\mathrm{D} 22}}\right)+\text { c.c. }\right]
\end{aligned}
$$

where we define $\Omega_{\mathrm{D} i i}=\operatorname{Re}\left[\Xi_{\mathrm{D} i i}\right]$ and $\Gamma_{\mathrm{D} i i}=-\operatorname{Im}\left[\Xi_{\mathrm{D} i i}\right]$. This result corresponds to the asymmetry from inverse decays while $\chi_{1}$ approaches equilibrium, when washout of the charge in $\varphi$ is ignored. When $\Delta M \gg \Gamma_{\mathrm{D}}$, this becomes

$$
S_{\varphi}=-2 \mathrm{i}\left(g_{1}^{2} g_{2}^{* 2}-g_{1}^{* 2} g_{2}^{2}\right) \int \frac{d^{3} q}{(2 \pi)^{3} 2 \sqrt{\mathbf{q}^{2}+\bar{M}^{2}}} \delta f_{0}(\mathbf{q}) \frac{\hat{\Pi}_{\chi}^{\mathcal{A}^{2}}}{M_{\chi_{11}}^{2}-M_{\chi_{22}}^{2}} \frac{\omega(\mathbf{q})}{\Pi_{\chi_{11}}^{\mathcal{A}}}
$$

The same answer can be obtained from integrating the source (23) for $\Gamma_{\mathrm{D}} \ll \Delta M$, when substituting $\delta f_{\chi_{i i}}=\delta f_{0} \exp \left(-\left(\Pi_{\chi_{11}}^{\mathcal{A}}(\omega(\mathbf{q}), \mathbf{q}) / \omega(\mathbf{q})\right) t\right)$. We can therefore conclude that indeed, oscillatory contributions to the $C P$-violating source approximately cancel in this regime.

Next, we compute the source (55) for the lepton asymmetry in a static background. For this purpose, we need to calculate the relevant components of the singlet neutrino propagator, $P_{\mathrm{R}} \mathrm{i} \delta S_{N i j}$. These can be expressed through the distribution functions (42), where the parameters $\Delta, D, \Gamma$ follow from Eqs. (32) and (777). As we ignore the effect of washout, we also neglect the lepton and Higgs chemical potentials when computing the singlet propagators and make use of the approximation (51). Notice that there are now two separate solutions for the helicity states $h= \pm$, and we can therefore identify the solutions (42) with $\delta f_{0 h}$, i.e. $\delta f_{0 h i j}(k)=\delta f_{i j}(k)$. The solutions thus following have the property $\delta f_{0 h i j}\left(k^{0}, \mathbf{k}\right)=\delta f_{0 h i j}^{*}\left(-k^{0}, \mathbf{k}\right)=\delta f_{0 h j i}\left(-k^{0}, \mathbf{k}\right)$, as a consequence of the Majorana condition (109) and the hermiticity (110) of the distribution functions. Making use of this relation, we can conveniently express the sum over positive and 
negative frequencies that enters into the source for the asymmetry (55) as

$$
\begin{aligned}
\mathcal{S}(\mathbf{k})=-Y_{i}^{*} Y_{j} \int \frac{d k^{0}}{2 \pi} \operatorname{tr}\left[P_{\mathrm{R}} \mathrm{i} \delta S_{N i j}(k) 2 P_{\mathrm{L}} \hat{\mathbb{E}}_{N}^{\mathcal{A}}(k)\right] \\
=Y_{i}^{*} Y_{j} \frac{1}{2} \sum_{\substack{k^{0}= \pm \omega(\mathbf{k}) \\
h= \pm}} \operatorname{tr}\left[P_{\mathrm{R}}\left(\gamma^{0}-h \hat{k}^{i} \gamma^{i}\right) \hat{\dot{E}}_{N}^{\mathcal{A}}(k)\right] \operatorname{sign}\left(k^{0}\right) \delta f_{0 h i j}\left(1+\frac{h|\mathbf{k}|}{k^{0}}\right) \\
=Y_{i}^{*} Y_{j} \sum_{h= \pm}\left\{\hat{\Sigma}_{N}^{\mathcal{A} 0}\left[\delta f_{0 h i j}-\delta f_{0 h i j}^{*}+\frac{h|\mathbf{k}|}{k^{0}}\left(\delta f_{0 h i j}+\delta f_{0 h i j}^{*}\right)\right]\right. \\
\left.-h \hat{k}^{i} \hat{\Sigma}_{N}^{\mathcal{A} i}\left[\delta f_{0 h i j}+\delta f_{0 h i j}^{*}+\frac{h|\mathbf{k}|}{k^{0}}\left(\delta f_{0 h i j}-\delta f_{0 h i j}^{*}\right)\right]\right\}_{k^{0}=\omega(\mathbf{k})} \\
=Y_{i}^{*} Y_{j} \sum_{h= \pm}\left\{\frac{k \cdot \hat{\Sigma}_{N}^{\mathcal{A}}}{k^{0}}\left(\delta f_{0 h i j}-\delta f_{0 h i j}^{*}\right)+h \frac{\tilde{k} \cdot \hat{\Sigma}_{N}^{\mathcal{A}}}{k^{0}}\left(\delta f_{0 h i j}+\delta f_{0 h i j}^{*}\right)\right\}_{k^{0}=\omega(\mathbf{k})}
\end{aligned}
$$

where we have used that $\hat{\Sigma}_{N}^{\mathcal{A} 0}\left(k^{0}, \mathbf{k}\right)=\hat{\Sigma}_{N}^{\mathcal{A} 0}\left(-k^{0}, \mathbf{k}\right)$ and $\hat{\Sigma}_{N}^{\mathcal{A} i}\left(k^{0}, \mathbf{k}\right)=-\hat{\Sigma}_{N}^{\mathcal{A}}\left(-k^{0}, \mathbf{k}\right)$. Above result for $\mathcal{S}(\mathbf{k})$ should be useful when $\delta f$ is calculated numerically, i.e. for phenomenological studies of Leptogenesis in the Early Universe, as we outline in Section 5 ,

When we neglect the expansion, we can again use the analytic solutions for damped flavour oscillations in Eqs. (42). Using Eqs. (77) and (32) and defining

$$
\delta \hat{f}_{12}=\delta f_{12} /\left(\mathrm{i} \Gamma_{12}\right),
$$

we obtain the relations

$$
\begin{gathered}
\delta f_{+012}(\omega(\mathbf{k}), \mathbf{k})=-\Xi_{12}\left(h=+, k^{0}=\omega(\mathbf{k})\right) \delta \hat{f}_{12}(\omega(\mathbf{k}), \mathbf{k}), \\
\delta f_{-012}(\omega(\mathbf{k}), \mathbf{k})=\Xi_{12}^{*}\left(h=+, k^{0}=\omega(\mathbf{k})\right) \delta \hat{f}_{12}(\omega(\mathbf{k}), \mathbf{k}), \\
\delta f_{+012}(-\omega(\mathbf{k}), \mathbf{k})=-\Xi_{12}^{*}\left(h=+, k^{0}=\omega(\mathbf{k})\right) \delta \hat{f}_{12}^{*}(\omega(\mathbf{k}), \mathbf{k}), \\
\delta f_{-012}(-\omega(\mathbf{k}), \mathbf{k})=\Xi_{12}\left(h=+, k^{0}=\omega(\mathbf{k})\right) \delta \hat{f}_{12}^{*}(\omega(\mathbf{k}), \mathbf{k}) .
\end{gathered}
$$

Note that in the mass-diagonal basis, $\Xi_{i j}=-\mathrm{i} \Gamma_{i j}$, for $i \neq j$. Above factorisation can easily be understood when noting that $\left|\Gamma_{12}\right|^{2}$, as given by Eq. (77), does not depend on helicity. Moreover, from Eqs. (32) and (77), it follows that $\Xi_{\mathrm{D}}$ does not depend on the helicity either. Therefore, the complete helicity dependence is isolated within the explicit front factor of $\Gamma_{12}$ in Eq. (42b). Substituting Eqs. (85) into Eq. (83), we obtain the simple expression 3

$$
\mathcal{S}(\mathbf{k})=\mathrm{i}\left(Y_{1}^{* 2} Y_{2}^{2}-Y_{1}^{2} Y_{2}^{* 2}\right) 2 g_{w} \frac{\bar{M}^{2}}{\mathbf{k}^{2}+\bar{M}^{2}} \hat{\Sigma}_{N \mu}^{\mathcal{A}} \hat{\Sigma}_{N}^{\mathcal{A} \mu}\left(\delta \hat{f}_{12}+\delta \hat{f}_{12}^{*}\right)
$$

\footnotetext{
${ }^{3}$ Note that the summation over $i, j=1,2$ that is understood in Eq. (83) by the sum convention is explicitly performed in the following equation.
} 

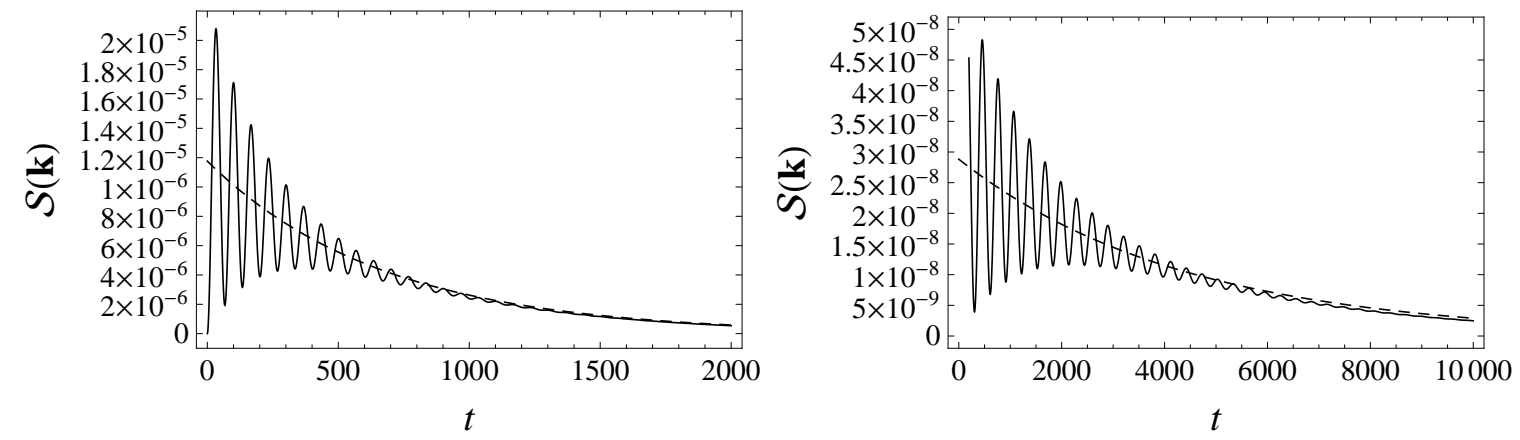

Figure 1: The source $\mathcal{S}(\mathbf{k})$ for the lepton asymmetry over time $t$ for $M_{1}=1, M_{2}=1.1$, $T=1, Y_{1}=0.1, Y_{2}=0.2+0.1$ i, i.e. $\Delta M \gg \Gamma_{\mathrm{D}}$, and initially a vanishing distribution for $N_{1}$ and an equilibrium distribution for $N_{2}$. The solid line is the result (83), the dashed line the standard perturbative limit (87). In the left panel, we take $|\mathbf{k}|=0.5$, and consider the asymmetry resulting from a non-relativistic singlet neutrino. In the right panel, the singlet neutrino is relativistic, $|\mathbf{k}|=5$.
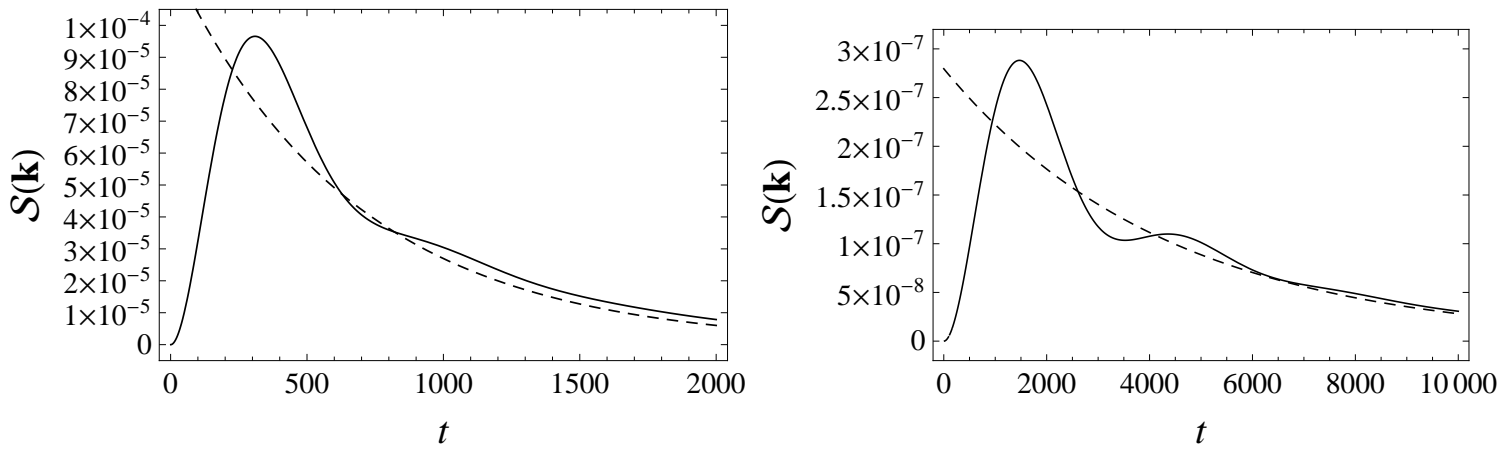

Figure 2: The source $\mathcal{S}(\mathbf{k})$ over time $t$ for the lepton asymmetry for $M_{1}=1, M_{2}=1.01$, $T=1, Y_{1}=0.1, Y_{2}=0.2+0.1$ i, i.e. $\Delta M \sim \Gamma_{\mathrm{D}}$, and initially a vanishing distribution for $N_{1}$ and an equilibrium distribution for $N_{2}$. The solid line is the result (83), the dashed line the standard perturbative limit (87). In the left panel, we take $|\mathbf{k}|=0.5$, and consider the asymmetry resulting from a non-relativistic singlet neutrino. In the right panel, the singlet neutrino is relativistic, $|\mathbf{k}|=5$.

This is the source for the lepton asymmetry that results from inverse decays of $N_{1}$ while it approaches the equilibrium distribution in a background of constant temperature. As a consistency check, the known result for $\Delta M \ll \bar{M}$ but $\Delta M \gg \Gamma_{\mathrm{D}}$ is recovered for $\delta \hat{f}_{12}=\delta f_{0}\left[\omega(\mathbf{k}) /\left(M_{11}^{2}-M_{22}^{2}\right)\right] \exp \left(-\Gamma_{11} t\right)$, which results from neglecting the oscillatory contributions in Eq. (42b).

We now compare the result from Eqs. (83) or (86) to the standard perturbative 
approximation

$$
\mathcal{S}(\mathbf{k})=\frac{8 \mathrm{i}\left[Y_{1}^{* 2} Y_{2}^{2}-Y_{1}^{2} Y_{2}^{* 2}\right]}{2 \sqrt{\mathbf{k}^{2}+\bar{M}^{2}}} \frac{M_{11} M_{22}}{M_{11}^{2}-M_{22}^{2}} g_{w} \hat{\Sigma}_{N \mu}^{\mathcal{A}} \hat{\Sigma}_{N}^{\mathcal{A} \mu} \mathrm{e}^{-2 g_{w}\left|Y_{1}\right|^{2}\left(k^{\mu} \hat{\Sigma}_{N \mu}^{\mathcal{A}} / k^{0}\right) t} \delta f_{0} .
$$

For this purpose, we use the solutions (42) with $\delta f_{0}(\mathbf{k})=-f^{\mathrm{eq}}(\mathbf{k})$, which corresponds to a vanishing number of $N_{1}$ at $t=0$ in the particular mode $\mathbf{k}$, while $N_{2}$ is in equilibrium. The other parameters are given in the Figure captions. Their choice does not correspond to a systematic study of parameter space, but the examples should be illustrative and representative. In Figure 1, we illustrate the situation when $\Delta M \gg \Gamma_{\mathrm{D}}$. The oscillation frequency is much larger than the damping rate, such that we may expect that these oscillations average out in the final result for the asymmetry, see the discussion above. We choose examples of non-relativistic and relativistic singlet neutrinos, because in the relativistic regime, the result turns out to be the remainder of an incomplete cancellation of larger terms involving $\hat{\Sigma}_{N}^{\mathcal{A} 0}$ and $\hat{\Sigma}_{N}^{\mathcal{A} i}$, which is therefore explicitly verified. The results in Figure 2 correspond to a point in parameter space where $\Delta M \sim \Gamma_{\mathrm{D}}$. Since the damping rate is now of the same order as the frequency of flavour oscillations between $N_{1}$ and $N_{2}$, these should not be neglected when calculating the asymmetry.

This brings us to comment on earlier expressions on the behaviour of the asymmetry when approaching the resonant regime. This matter is discussed in Refs. [4, 8, 9, 47], where expressions for the asymmetries are given. Oscillations are neglected there, an approximation that is not always suitable, $c f$. Figure 2. It is however emphasised in Refs. [4, 8, 9, 47], that in the regimes $\Delta M \sim \Gamma_{\mathrm{D}}$ and $\Delta M \ll \Gamma_{\mathrm{D}}$, the standard perturbative result breaks down and a suitable resummation technique for the singlet neutrino propagator should be employed, which is essentially what is performed within the present work. It has already been mentioned in Section 2.3, that when choosing $U \delta f(0) U^{\dagger}$ as a diagonal matrix, there are no flavour oscillations. It should however be emphasised that this corresponds to a peculiar initial state that already bears a $C P$ asymmetry. From Eqs. (32) and (86) it then follows that

$$
\mathcal{S}(\mathbf{k})=\mathrm{i}\left(Y_{1}^{* 2} Y_{2}^{2}-Y_{1}^{2} Y_{2}^{* 2}\right) 2 g_{w} \frac{\bar{M}}{\mathbf{k}^{2}+\bar{M}^{2}} \hat{\Sigma}_{N \mu}^{\mathcal{A}} \hat{\Sigma}_{N}^{\mathcal{A} \mu}\left(\frac{1}{\Delta+D}+\frac{1}{\Delta^{*}+D^{*}}\right) \delta f_{0 h i i} .
$$

Notice that $\Delta+D \neq 0$, as long as $Y_{i} \neq 0$. This result is in agreement with Refs. [45, 46], where the time evolution of mixing scalars in the vacuum is considered. It generalises the findings of Refs. [45, 46] to mixing Majorana fermions, that decay in a finite-temperature background. In the limit $\Delta M \gg \Gamma_{\mathrm{D}}$, the term $\sim \Xi_{12} \Xi_{21}$ in the discriminant of $D$ can be neglected, and the results of Refs. [4, 47] for Majorana neutrino decay in a zero temperature background is recovered. We emphasise however, that when the condition $\Delta M \gg \Gamma_{\mathrm{D}}$ does not apply, the full time-evolution of the off-diagonal components of the singlet neutrino propagator has to be calculated, because due to oscillation effects, these are not simply proportional to the diagonal components in general.

Finally, let us briefly discuss the limit $\left|\Gamma_{12}\right| \ll\left|\Gamma_{11}-\Gamma_{22}\right|$ and $\left|\Gamma_{12}\right| \lesssim \Delta M$ in order to facilitate the comparison with the results in Ref. [72]. In this limit the term $\sim \Xi_{12} \Xi_{21}$ in 
the discriminant of $D$ can again be neglected, and Eq. (866) for the $C P$-violating source reduces to

$$
\begin{aligned}
\mathcal{S}(\mathbf{k})= & \frac{8 \mathrm{i}\left[Y_{1}^{* 2} Y_{2}^{2}-Y_{1}^{2} Y_{2}^{* 2}\right]}{2 \sqrt{\mathbf{k}^{2}+\bar{M}^{2}}} \frac{M_{11} M_{22}\left(M_{11}^{2}-M_{22}^{2}\right)}{\left(M_{11}^{2}-M_{22}^{2}\right)^{2}+\omega^{2}\left(\Gamma_{11}-\Gamma_{22}\right)^{2}} g_{w} \hat{\Sigma}_{N \mu}^{\mathcal{A}} \hat{\Sigma}_{N}^{\mathcal{A} \mu} \\
& \times\left[\mathrm{e}^{-\Gamma_{11} t}-\left(\cos (\Delta \omega t)+\frac{\Gamma_{11}-\Gamma_{22}}{2 \Delta \omega} \sin (\Delta \omega t)\right) \mathrm{e}^{-\left(\Gamma_{11}+\Gamma_{22}\right) t / 2}\right] \delta f_{0} .
\end{aligned}
$$

where $\Delta \omega(\mathbf{k}) \equiv\left|M_{11}^{2}-M_{22}^{2}\right| /(2 \omega(\mathbf{k}))$. This result is found to be in agreement with the analytical results presented in Ref. [72] 44 Furthermore, the structure of the denominator $\left(M_{11}^{2}-M_{22}^{2}\right)^{2}+\omega^{2}\left(\Gamma_{11}-\Gamma_{22}\right)^{2}$ agrees with the results in ref. [47]. However, we want to emphasize that the parametric regime with $\left|\Gamma_{12}\right| \ll\left|\Gamma_{11}-\Gamma_{22}\right|$ is by no means generic in resonant leptogenesis.

\section{$5 \quad$ Effective Theory in the Expanding Universe}

In this Section, we present a network of equations that describes the simplest version of resonant Leptogenesis in the expanding Universe. Equations that are valid sufficiently far away from the resonant limit, i.e. for $\Delta M \gg \Gamma_{\mathrm{D}}$, are presented in Ref. [33]. In the present context, we need to generalise Eq. (174) in such a way, that it encompasses the effect of the expanding background, in particular how this induces a deviation of the distribution functions of the singlet neutrinos from equilibrium.

First, we transform the time coordinate $t$ to the conformal time $\eta$ through the relation $d t=a(\eta) d \eta$, where $a(\eta)$ denotes the scale factor. In the radiation dominated Universe, $a(\eta)=a_{\mathrm{R}} \eta$, where $a_{\mathrm{R}}$ is a constant. The scale factor is related to the physical temperature by

$$
T=\frac{1}{a_{\mathrm{R}} \eta} \sqrt{\frac{a_{\mathrm{R}} m_{\mathrm{Pl}}}{2}}\left(\frac{45}{\pi^{3} g_{*}}\right)^{1 / 4},
$$

where $g_{*}$ denotes the number of relativistic degrees of freedom. Note that the comoving temperature $T_{\text {com }}=a(\eta) T$ is constant. There remains a freedom of parametrisation for $a_{\mathrm{R}}$. For kinetic equations in the early Universe, $z=\bar{M} \eta$ is a convenient choice, where

$$
z=\frac{\bar{M}}{T},
$$

and therefore

$$
a_{\mathrm{R}}=\frac{m_{\mathrm{Pl}}}{2} \sqrt{\frac{45}{\pi^{3} g_{*}}} .
$$

\footnotetext{
${ }^{4}$ Note that in Ref. [72] the initial state corresponds to vanishing number densities for both neutrino flavours $N_{1}$ and $N_{2}$. Furthermore, the analytical results in [72] assume the non-relativistic limit $M_{i} \gg T$, such that $\hat{\Sigma}_{N \mu}^{\mathcal{A}}(k) \approx k^{\mu} /(32 \pi)$.
} 
The choice of the conformal time $\eta$ instead of the comoving proper time $t$ has the advantage that the Kadanoff Baym equations are of a form that is very similar to the one they take in Minkowski background. The only modification is that the mass terms need to be rescaled as $M \rightarrow a(\eta) M$ and the momenta are to be understood as comoving momenta. The derivative with respect to $\eta$ [or, equivalently, $z / \bar{M}$, when using Eq. (92)] is denoted by a prime.

Using these parametrisations, one can obtain a term that represents the deviation of the singlet neutrino from equilibrium, that is induced by the expansion of the Universe. When $\Delta M \ll \bar{M}$, the equilibrium distribution for the singlet neutrinos is

$$
f_{N i i}^{\mathrm{eq}}(\eta)=\frac{1}{\mathrm{e}^{\sqrt{\mathbf{k}^{2}+a^{2}(\eta) \bar{M}^{2}} / T_{\mathrm{com}}+1}}
$$

and $f_{N i j}^{\mathrm{eq}}(z)=0$ for $i \neq j$. When we are not working in the mass eigenbasis, this relation is only approximately correct and there will be off-diagonal elements that are suppressed by a factor of $\Delta M / M$ when compared to the diagonal ones. While this is a tolerable error, it could easily be corrected for by diagonalising $M$ through an orthogonal transformation. The expanding background induces a decrease of the temperature, such that consequently,

$$
\frac{d}{d \eta} f_{N i i}^{\mathrm{eq}}(\eta)=f_{N i i}^{\mathrm{eq} \prime}(\eta)=-\frac{\mathrm{e}^{\sqrt{\mathbf{k}^{2}+a^{2}(\eta) \bar{M}^{2}} / T_{\mathrm{com}}}}{\left(\mathrm{e}^{\sqrt{\mathbf{k}^{2}+a^{2}(\eta) \bar{M}^{2}} / T_{\mathrm{com}}}+1\right)^{2}} \frac{a_{\mathrm{R}}^{2} \bar{M}^{2} \eta}{T_{\mathrm{com}} \sqrt{\mathbf{k}^{2}+a^{2}(\eta) \bar{M}^{2}}}
$$

and $f_{N i j}^{\mathrm{eq} \prime}(z)=0$ for $i \neq j$.

The generalisation of Eq. (174), valid in Minkowski background, to an expanding, radiation dominated Universe is now given by

$$
\begin{aligned}
\delta f_{0 h}^{\prime}+a^{2}(\eta) \frac{1}{2 k^{0}} \mathrm{i}\left[M^{2}, \delta f_{0 h}\right]+f^{\mathrm{eq} \prime}= & -\left(\Sigma_{N \mathrm{~L}}^{\mathcal{A 0}}+h \hat{k}^{i} \Sigma_{N \mathrm{~L}}^{\mathcal{A} i}\right)\left(1-\frac{h|\mathbf{k}|}{k^{0}}\right)\left\{\mathbf{Y}^{*} \mathbf{Y}^{t}, \delta f_{0 h}\right\} \\
& -\left(\Sigma_{N \mathrm{~A}}^{\mathcal{A} 0}-h \hat{k}^{i} \Sigma_{N \mathrm{R}}^{\mathcal{A} i}\right)\left(1+\frac{h|\mathbf{k}|}{k^{0}}\right)\left\{\mathbf{Y Y}^{\dagger}, \delta f_{0 h}\right\} \\
\text { where } k^{0}= & \pm \sqrt{\mathbf{k}^{2}+a(\eta)^{2} \bar{M}^{2}}
\end{aligned}
$$

Note that this equation makes no assumption about the form of $M$, except that it is a real symmetric matrix, which allows to account for temperature-dependent effective masscorrections that may be incurred through $\mathbb{K}_{N}^{H}$. Eq. (95) is therefore flavour-covariant with respect to orthogonal transformations of $N_{i}$. Allowing for a complex symmetric $M$ would lead to a more complicated form of Eq. (95), but it would not capture addtional physical parameter space, as the complex phases in $M$ can always be absorbed in $Y$, cf. the discussion in Section 3.1. Therefore, Eq. (95) can be used also in situations where the mass eigenbasis is time-dependent due to finite-temperature effects. This should be 
of importance close to the resonant limit, since the thermal corrections to $M$ that arise from $\not_{N}^{H}$ are given by (for momenta $|\mathbf{k}| \gg\left|M_{i j}^{\mathrm{Th}}\right|$, which covers the relevant contributions of phase-space)

$$
M_{i j}^{\mathrm{Th}}{ }^{2}=\frac{1}{4}\left(Y_{i}^{*} Y_{j}+Y_{i} Y_{j}^{*}\right) T^{2}
$$

which is of the same order as $k^{0} \Gamma_{\mathrm{D}}$.

Assuming that the lepton charge density is small compared to the number density (or, equivalently, the lepton chemical potential $\mu_{\ell}$ is small compared to the temperature T), we extract from Eq. (54) the washout term as

$$
\begin{aligned}
W & =Y_{i}^{*} Y_{j} \int \frac{d^{4} k}{(2 \pi)^{4}} \int \frac{d^{4} k^{\prime}}{(2 \pi)^{4}} \int \frac{d^{4} k^{\prime \prime}}{(2 \pi)^{4}}(2 \pi)^{4} \delta^{4}\left(k-k^{\prime}-k^{\prime \prime}\right) \\
& \times \operatorname{tr}\left[\left(P_{\mathrm{R}} \mathrm{i} S_{N i j}^{>}\left(k^{\prime}\right) P_{\mathrm{L}} \mathrm{i} \Delta_{\phi}^{<}\left(-k^{\prime \prime}\right)-P_{\mathrm{R}} \mathrm{i} S_{N i j}^{<}\left(k^{\prime}\right) P_{\mathrm{L}} \mathrm{i} \Delta_{\phi}^{>}\left(-k^{\prime \prime}\right)\right) \mathrm{i} \delta S_{\ell}(k)\right] .
\end{aligned}
$$

The deviation of the lepton propagator $\mathrm{i} \delta S_{\ell}(k)$ from the form with vanishing chemical potential can be expressed through

$$
\delta f_{\ell}(\mathbf{k})=-\delta \bar{f}_{\ell}(\mathbf{k})=\left(n_{\ell}-\bar{n}_{\ell}\right) \times \frac{6 \mathrm{e}^{|\mathbf{k}| / T}}{T^{3}\left(\mathrm{e}^{|\mathbf{k}| / T}+1\right)^{2}}=\mu_{\ell} \frac{\mathrm{e}^{|\mathbf{k}| / T}}{T\left(\mathrm{e}^{|\mathbf{k}| / T}+1\right)^{2}},
$$

where

$$
\mathrm{i} \delta S_{\ell}(k)=-P_{\mathrm{L}} \not k 2 \pi \delta\left(k^{2}\right) \delta f_{\ell}(\mathbf{k}) \operatorname{sign}\left(k^{0}\right) .
$$

In contrast to the washout term quoted in Ref. [33], we include here the off-diagonal elements of the singlet neutrino propagator. When $\Delta M \gg \Gamma_{\mathrm{D}}$, these are suppressed compared to the out-of-equilibrium components by a factor of $\Gamma_{\mathrm{D}} / \Delta M$, i.e. we can safely neglect these in the non-resonant regime. When $\Delta M$ is of order $\Gamma_{\mathrm{D}}$ or smaller, neglecting the off-diagonal correlations is not necessarily a good approximation. Especially in the weak washout regime $\delta f_{0 h}$ may be of similar size as $f^{\mathrm{eq}}$, and these corrections may be of importance. In the strong washout regime however, the deviation of the singlet neutrino density from equilibrium is small, such that we can substitute the equilibrium propagator for $i S_{N}$ in Eq. (97), which is diagonal in the mass eigenbasis, when neglecting finite-width contributions.

The collision term on the right-hand-side of Eq. (95) is valid when the chemical potentials of the Higgs and lepton fields can be neglected. Once this is not the case, it 
should be replaced with

$$
\begin{aligned}
& -\frac{\mathrm{i}}{2}\left(\Sigma_{N \mathrm{~L}}^{>0}+h \hat{k}^{i} \Sigma_{N \mathrm{~L}}^{>i}\right)\left(1-\frac{h|\mathbf{k}|}{k^{0}}\right)\left\{\mathbf{Y}^{*} \mathbf{Y}^{t}, f_{0 h}\right\} \\
& -\frac{\mathrm{i}}{2}\left(\Sigma_{N \mathrm{R}}^{>0}-h \hat{k}^{i} \Sigma_{N \mathrm{R}}^{>i}\right)\left(1+\frac{h|\mathbf{k}|}{k^{0}}\right)\left\{\mathbf{Y} \mathbf{Y}^{\dagger}, f_{0 h}\right\} \\
& +\frac{\mathrm{i}}{2}\left(\Sigma_{N \mathrm{~L}}^{<0}+h \hat{k}^{i} \Sigma_{N \mathrm{~L}}^{<i}\right)\left(1-\frac{h|\mathbf{k}|}{k^{0}}\right)\left\{\mathbf{Y}^{*} \mathbf{Y}^{t}, 1+f_{0 h}\right\} \\
& +\frac{\mathrm{i}}{2}\left(\Sigma_{N \mathrm{R}}^{<0}-h \hat{k}^{i} \Sigma_{N \mathrm{R}}^{<i}\right)\left(1+\frac{h|\mathbf{k}|}{k^{0}}\right)\left\{\mathbf{Y} \mathbf{Y}^{\dagger}, 1+f_{0 h}\right\} .
\end{aligned}
$$

Here, $f_{0 h}=f_{0 h}^{\mathrm{eq}}+\delta f_{0 h}$ is the full distribution matrix of $N$, that also includes the equilibrium distribution $f_{0 h}^{\mathrm{eq}}(\mathbf{k})=\operatorname{diag}\left(1 /\left(\exp \left(\sqrt{\mathbf{k}^{2}+M_{11}^{2}} / T\right)+1\right), 1 /\left(\exp \left(\sqrt{\mathbf{k}^{2}+M_{22}^{2}} / T\right)+1\right)\right)$. The $\Sigma_{N}^{<,>}$can be obtained from the expressions for $\Sigma_{N}^{\mathcal{A}}$ in Appendix $\mathrm{B}$ when using the Kubo-Martin-Schwinger (KMS) relations

$$
\Sigma_{N \mathrm{~L}, \mathrm{R}}^{>}(k)=-\mathrm{e}^{\left(k^{0} \mp \mu_{\ell} \mp \mu_{\phi}\right) / T} \Sigma_{N \mathrm{~L}, \mathrm{R}}^{>}(k) .
$$

It should be useful to expand this term in the small ratios $\mu_{\ell, \phi} / T$, in order to separate it into a contribution of the form as in Eq. (95) and an additional one, that only depends on the chemical potentials. A detailed study of this matter is subject of ongoing work.

Of course, Eq. (54) must be transformed from comoving to conformal time as well, with the result

$$
\frac{d}{d \eta}\left(n_{\ell}-\bar{n}_{\ell}\right)=W+S
$$

For $\Delta M \gg \Gamma_{\mathrm{D}}$, the procedure of solving for the lepton asymmetry is described in detail in Ref. [33]. We first determine $\delta f_{0 h i j}(k)$ from Eq. (95) and the approximation (51) of vanishing chemical potentials for Higgs and leptons. The result is then to be substituted into the source term (55) and the washout term (97). When $\Delta M \lesssim \Gamma_{\mathrm{D}}$, we should not expect the approximation (51) substituted into the kinetic equations for the oscillating singlet neutrinos (95) to be sufficient, as it may fail to accurately describe the washout of the lepton asymmetry. In that situation, the evolution of the lepton asymmetry (102) and of the correlation of the singlet neutrinos (95) should be solved as a coupled set of equations. In principle, the lepton and the Higgs chemical potentials are further processed by spectator effects. A good approximation for first phenomenological studies may be to consider an unflavoured regime, where the charge in $\ell$ is conserved, up to the interactions with $N_{i}$, that are accounted for already by our equations. The hypercharge asymmetry within $\phi$ should get distributed over a large number of remaining Standard Model degrees of freedom, such that we may set $\mu_{\phi} \approx 0$ within a first approximation.

Note that for the source term, the form given by Eq. (83) may be particularly useful, because we only have to calculate the positive frequency solutions for $\delta f_{0 h i j}(k)$, while 
the negative frequency solution follows as a consequence of the Majorana condition. Eventually, Eqs. (95) and (102) can be integrated in order to obtain the final lepton asymmetry. A numerical study of the evolution of the lepton asymmetry, using the methods that are outlined in this Section, should be performed in future work. In particular, when $\Delta M \gg \Gamma_{\mathrm{D}}$, it appears plausible that for an equilibrium deviation induced by the expanding background, the oscillating contributions visible in Figure 1 vanish altogether, because the deviation of the singlet neutrinos from equilibrium is generated continuously throughout Leptogenesis. Deviations that appear at different times oscillate and have different phases, such that the oscillations may effectively vanish due to decoherence. However, when $\Delta M \approx \Gamma_{\mathrm{D}}$ or $\Delta M \ll \Gamma_{\mathrm{D}}$, the methods outlined in this Section may be suitable to make predictions for resonant Leptogenesis that are not accurately covered by the perturbative result (68) for the source term.

\section{Summary and Conclusions}

In this paper, we have developed the theory of Leptogenesis from mixing Majorana fermions and from mixing scalars in the CTP formalism. Our calculations rely on the expansion parameters $\Gamma_{\mathrm{D}} \ll \Delta M$ or $\Delta M \ll \bar{M}$. Due to the smallness of the couplings, $\Gamma_{\mathrm{D}} \ll \bar{M}$, such that the parametric regimes where the two approximations apply overlap. When $\Gamma_{\mathrm{D}} \ll \Delta M$, the usual result for the $C P$-asymmetry from the wave-function correction applies [3, 6 -10, 33]. Otherwise, oscillations are relevant for the $C P$ asymmetry, and it is necessary to solve the kinetic equations for the singlet neutrino propagator. In Section 5, we explain how such a solution may be obtained when taking the expansion of the Universe into account and how this solution can be used to calculate the final value of the lepton asymmetry of the Universe.

Within our results, we recover many elements that have previously been derived using Boltzmann equations and $S$-matrix elements or the Hamiltonian approach. However, there are a few features that differ from the earlier approaches:

- For Leptogenesis from the decays of Majorana fermions, we show that the kinetic evolution equations (26) can accurately reproduce the classic result [3, 66 10, 33] for the asymmetry in Resonant Leptogenesis (68). This requires a detailed analytical computation of the evolution of the two helicity states of the singlet neutrinos, and has not been provided in earlier work based on the Hamiltonian approach [24 26].

- Our results consistently include the quantum statistical (Fermi-Dirac and BoseEinstein) corrections that appear for all on-shell particles in the finite-density background. Note that these also apply to the on-shell particles within the loops of the diagrams that describe $C P$-violation.

- When $\Delta M \sim \Gamma_{\mathrm{D}}$ or smaller, it is important to take account of the lepton and Higgs chemical potentials, because in this situation, the lepton number violation 
by the singlet neutrinos proceeds so slowly, that we have to take account of its timedependence. This may be crucial in order to treat the washout of the asymmetry in a quantitatively correct manner.

- Our effective theory is formulated within one single framework, the CTP formalism. In contrast, earlier approaches typically combine elements from $S$-matrices, classical Boltzmann equations or Hamiltonian transition amplitudes. In particular, in combination with Ref. [34], the methods presented here cover both, direct $C P$-violation and $C P$-violation from mixing, even when oscillations are important. Working within a single formalism may have the advantage, that it is easier to implement controlled approximations. The CTP approach combines the advantage of the $S$-matrix approach, that perturbative expansions can be efficiently formulated in terms of Feynman diagrams, with the advantage of the Hamiltonian real-time evolution, that avoids unitarity violation from the overcounting of real intermediate states.

- Since the CTP approach is formulated in terms of Green functions and Feynman diagrams, this method for calculating the lepton asymmetry may be particularly suitable to be combined with corrections due to scattering processes in the thermal bath [38-44].

The CTP approach appears as a well-suited method for a consistent description of macroscopic statistical systems that consist of High Energy Particle Physics degrees of freedom. In particular, it may provide an intuitive and accurate framework in order to describe the emergence of the baryon asymmetry of the Universe from the quantum effect of $C P$-violation. Further significant progress on the theory of Leptogenesis appears to be a realistic prospect. For Resonant Leptogenesis, the present work is intended to pave the way to improve the quantitative predictions and the qualitative understanding.

\section{Additional Notes}

Another successful calculation of the asymmetry for Resonant Leptogenesis in the CTP approach, using partly different calculational strategies than those in the present work, was reported by Garny et al. [72]. During the final stage of the preparation of this manuscript, Ref. [73] appeared. Based on the Hamiltonian approach, it is also pointed out there, that the lepton chemical potential is relevant for computing the correlations of the singlet neutrino flavours.

\section{Acknowledgements}

This work is supported by the Gottfried Wilhelm Leibniz programme of the Deutsche Forschungsgemeinschaft and by the Alexander von Humboldt Foundation. 


\section{A Two-Point Functions on the CTP}

Two-point functions on the CTP are endowed with two path indices $+/-$. We often make use of the definitions

$$
\begin{aligned}
& G^{++}(x, y)=G^{T}(x, y), \\
& G^{+-}(x, y)=G^{<}(x, y), \\
& G^{-+}(x, y)=G^{>}(x, y), \\
& G^{--}(x, y)=G^{\bar{T}}(x, y),
\end{aligned}
$$

where $G$ stands for any two-point function, i.e. fermionic or scalar, or Green function or propagator.

The propagators of a scalar field $\chi$ are

$$
\begin{aligned}
& \mathrm{i} \Delta^{T}(x, y)=\left\langle T\left[\chi(x) \chi^{\dagger}(y)\right]\right\rangle, \\
& \mathrm{i} \Delta^{<}(u, v)=\left\langle\chi^{\dagger}(y) \chi(x)\right\rangle, \\
& \mathrm{i} \Delta^{>}(x, y)=\left\langle\chi(x) \chi^{\dagger}(y)\right\rangle, \\
& \mathrm{i} \Delta^{\bar{T}}(x, y)=\left\langle\bar{T}\left[\chi(x) \chi^{\dagger}(y)\right]\right\rangle .
\end{aligned}
$$

In order to describe mixing of flavours, $\chi$ ought to be considered as a column vector in flavour space. $T(\bar{T})$ stands for (anti-)time ordering and the superscript for the corresponding Green functions. We sometimes refer to the Green functions with the superscripts $<,>$ as Wightman functions. When $\chi$ is real, the neutrality constraint $\chi^{c}=\chi^{\dagger}=\chi$ must be observed. It then follows that

$$
\Delta(x, y)=\Delta^{t}(y, x),
$$

where the superscript $t$ denotes the transposition which acts here on both, CTP $( \pm)$

and flavour indices. For both, real and complex fields the Wightman functions have the hermiticity property

$$
\mathrm{i} \Delta^{<,>}(x, y)=\left(\mathrm{i} \Delta^{<,>}(y, x)\right)^{\dagger} .
$$

Similarly, for a fermion $\psi$, the Green functions are

$$
\begin{aligned}
& \mathrm{i} S^{T}(x, y)=\langle T[\psi(x) \bar{\psi}(y)]\rangle, \\
& \mathrm{i} S^{<}(x, y)=-\langle\bar{\psi}(y) \psi(x)\rangle, \\
& \mathrm{i} S^{>}(x, y)=\langle\psi(x) \bar{\psi}(y)\rangle, \\
& \mathrm{i} S^{\bar{T}}(x, y)=\langle\bar{T}[\psi(x) \bar{\psi}(y)]\rangle .
\end{aligned}
$$


Notice that these define matrices in Spinor space. A Majorana spinor mus observe the condition

$$
\psi^{c}=C \bar{\psi}^{t}=\psi
$$

where $C$ is the charge conjugation matrix. As a consequence, for Majorana two-point functions,

$$
S(x, y)=C S^{t}(y, x) C^{\dagger}
$$

where the transposition acts on all, CTP $( \pm)$, spinor and flavour indices. The hermiticity property of the Wightman function reads now

$$
\mathrm{i} \gamma^{0} S^{<,>}(x, y)=\left(\mathrm{i} \gamma^{0} S^{<,>}(y, x)\right)^{\dagger}
$$

which holds for both, charged and Majorana spinors.

Additional useful two-point functions follow when taking the combinations

$$
\begin{aligned}
& G^{A}=G^{T}-G^{>}=G^{<}-G^{\bar{T}} \quad(\text { advanced }), \\
& G^{R}=G^{T}-G^{<}=G^{>}-G^{\bar{T}} \quad(\text { retarded }), \\
& G^{H}=\frac{1}{2}\left(G^{R}+G^{A}\right) \quad(\text { Hermitian }), \\
& G^{\mathcal{A}}=\frac{1}{2 \mathrm{i}}\left(G^{A}-G^{R}\right)=\frac{\mathrm{i}}{2}\left(G^{>}-G^{<}\right) \quad(\text { anti-Hermitian, spectral }) .
\end{aligned}
$$

The Wigner transform of a two-point function is defined as

$$
G(k, x)=\int d^{4} r \mathrm{e}^{\mathrm{i} k r} G(x+r / 2, x-r / 2),
$$

where we refer to $r$ as the relative and to $x$ as the average coordinate. In the situation of spatial homogeneity, there is no dependence on $\mathbf{x}$ (spatial translation invariance), such that $G(k, x) \equiv G(k, t)$.

The transformation of convolution integrals in coordinate space into Wigner space can be expressed with the help of the diamond operator, which is defined as

$$
\diamond\{A\}\{B\}=\frac{1}{2}\left(\partial_{x} A\right)\left(\partial_{k} B\right)-\frac{1}{2}\left(\partial_{k} A\right)\left(\partial_{x} B\right)
$$

where $A \equiv A(k, x)$ and $B \equiv B(k, x)$.

\section{B Equilibrium Self-Energies}

The spectral self-energy, that is central in calculations of the resonantly generated lepton asymmetry is computed in Ref. [33]. Here, we generalise that expression in order to 
account for the lepton and Higgs chemical potentials. This may be of importance when $\Delta M \gg \Gamma_{\mathrm{D}}$ is not valid, such that lepton number violation is modulated by the slow oscillations of the right-handed neutrinos. Neglecting tree-level and thermal masses for the lepton and the Higgs boson, the normalised spectral self-energy can be expressed as

$$
\begin{aligned}
\hat{\Sigma}_{N \mathrm{~L}, \mathrm{R}}^{\mathcal{A} 0}(k) & =\frac{T^{2}}{16 \pi|\mathbf{k}|}\left[\vartheta\left(k^{0}\right) I_{1}\left(\frac{\left|k^{0}\right|}{T}, \frac{|\mathbf{k}|}{T}, \mp \frac{\mu_{\ell}}{T}, \mp \frac{\mu_{\phi}}{T}\right)+\vartheta\left(-k^{0}\right) I_{1}\left(\frac{\left|k^{0}\right|}{T}, \frac{|\mathbf{k}|}{T}, \pm \frac{\mu_{\ell}}{T}, \pm \frac{\mu_{\phi}}{T}\right)\right], \\
\hat{\Sigma}_{N \mathrm{~L}, \mathrm{R}}^{\mathcal{A} i}(k) & =\frac{T^{2} \hat{k}^{i}}{16 \pi|\mathbf{k}|} \\
& \times\left[\vartheta\left(k^{0}\right)\left(\frac{\left|k^{0}\right|}{|\mathbf{k}|} I_{1}\left(\frac{\left|k^{0}\right|}{T}, \frac{|\mathbf{k}|}{T}, \mp \frac{\mu_{\ell}}{T}, \mp \frac{\mu_{\phi}}{T}\right)-\frac{M_{1}^{2}}{2|\mathbf{k}| T} I_{0}\left(\frac{\left|k^{0}\right|}{T}, \frac{|\mathbf{k}|}{T}, \mp \frac{\mu_{\ell}}{T}, \mp \frac{\mu_{\phi}}{T}\right)\right)\right. \\
& \left.-\vartheta\left(-k^{0}\right)\left(\frac{\left|k^{0}\right|}{|\mathbf{k}|} I_{1}\left(\frac{\left|k^{0}\right|}{T}, \frac{|\mathbf{k}|}{T}, \pm \frac{\mu_{\ell}}{T}, \pm \frac{\mu_{\phi}}{T}\right)-\frac{M_{1}^{2}}{2|\mathbf{k}| T} I_{0}\left(\frac{\left|k^{0}\right|}{T}, \frac{|\mathbf{k}|}{T}, \pm \frac{\mu_{\ell}}{T}, \pm \frac{\mu_{\phi}}{T}\right)\right)\right],
\end{aligned}
$$

where

$$
I_{n}\left(y_{0}, y, \varrho_{\ell}, \varrho_{\phi}\right) \equiv \int_{\frac{1}{2}\left(y_{0}-y\right)}^{\frac{1}{2}\left(y_{0}+y\right)} d x x^{n}\left(1-\frac{1}{\mathrm{e}^{x-\varrho_{\ell}}+1}+\frac{1}{\mathrm{e}^{y_{0}-x-\varrho_{\phi}}-1}\right)
$$

These integrals are given by

$$
\begin{aligned}
I_{0}\left(y_{0}, y\right)= & {\left[\log \left(\mathrm{e}^{x}-\mathrm{e}^{\varrho \ell}\right)-\log \left(\mathrm{e}^{x+\varrho_{\phi}}-\mathrm{e}^{y^{0}}\right)\right]_{x=\frac{1}{2}\left(y^{0}-y\right)}^{x=\frac{1}{2}\left(y^{0}+y\right)}, } \\
I_{1}\left(y_{0}, y\right)= & {\left[x\left(\log \left(1+\mathrm{e}^{x-\varrho \ell}\right)-\log \left(1-\mathrm{e}^{x-y^{0}+\varrho_{\phi}}\right)\right)\right.} \\
& \left.+\operatorname{Li}_{2}\left(-\mathrm{e}^{x-\varrho_{\ell}}\right)-\operatorname{Li}_{2}\left(\mathrm{e}^{x-y^{0}+\varrho_{\phi}}\right)\right]_{x=\frac{1}{2}\left(y^{0}-y\right)}^{x=\frac{1}{2}\left(y^{0}+y\right)}
\end{aligned}
$$

where $\mathrm{Li}_{2}$ is the dilogarithm. Of course, for $\mu_{\ell}=\mu_{\phi}=0, \hat{\Sigma}_{N \mathrm{~L}}^{\mathcal{A} \mu}=\hat{\Sigma}_{N \mathrm{R}}^{\mathcal{A} \mu}$.

For the scalar model, we do not use the explicit form of the equilibrium self-energies for the studies presented in this paper. For completeness, we note that these can be calculated by defining

$$
p_{ \pm}^{0}=\frac{1}{2 k^{2}}\left\{k^{0}\left(k^{2}+m_{a}^{2}-m_{b}^{2}\right) \pm \sqrt{\mathbf{k}^{2}\left(k^{2}+m_{a}^{2}-m_{b}^{2}\right)^{2}-4 \mathbf{k}^{2} k^{2} m_{a}^{2}}\right\}
$$


and

$$
\begin{aligned}
& P\left(k, G, m_{a} \cdot m_{b}, s_{a}, s_{b}, L_{f}\right) \\
& \quad=G \frac{k^{2}}{|\mathbf{k}|} \operatorname{sign}^{L_{f}}\left(k^{2}\right)\left\{\vartheta\left(-k^{2}\right) k^{0}-p_{+}^{0}+p_{-}^{0}+\frac{1}{\beta} \log \left|\frac{\mathrm{e}^{\beta p_{+}^{0}}-s_{a}}{\mathrm{e}^{\beta p_{-}^{0}}-s_{a}}\right|-\frac{1}{\beta} \log \left|\frac{\mathrm{e}^{\beta\left(k^{0}-p_{+}^{0}\right)}-s_{b}}{\mathrm{e}^{\beta\left(k^{0}-p_{-}^{0}\right)}-s_{b}}\right|\right\}
\end{aligned}
$$

for $k^{2} \geq 0$.

The equilibrium spectral self-energy for the neutral scalar field $\chi$ is then given by

$$
\Pi_{\chi_{i j}}^{\mathcal{A}}(k)=P\left(k, \frac{g_{1} g_{2}^{*}+g_{1}^{*} g_{2}}{16 \pi}, m_{\varphi}, m_{\varphi},+1,+1,0\right) \text {. }
$$

\section{Green Functions for Leptogenesis}

The propagators for the leptons are given by

$$
\begin{aligned}
& \mathrm{i} S_{\ell}^{<}(p)=-2 \pi \delta\left(p^{2}\right) P_{\mathrm{L}} \not P_{\mathrm{R}}\left[\vartheta\left(p_{0}\right) f_{\ell}(\mathbf{p})-\vartheta\left(-p_{0}\right)\left(1-\bar{f}_{\ell}(-\mathbf{p})\right)\right], \\
& \mathrm{i} S_{\ell}^{>}(p)=-2 \pi \delta\left(p^{2}\right) P_{\mathrm{L}} \not P_{\mathrm{R}}\left[-\vartheta\left(p_{0}\right)\left(1-f_{\ell}(\mathbf{p})\right)+\vartheta\left(-p_{0}\right) \bar{f}_{\ell}(-\mathbf{p})\right], \\
& \mathrm{i} S_{\ell}^{T}(p)=P_{\mathrm{L}} \frac{\mathrm{i} p}{p^{2}+\mathrm{i} \varepsilon} P_{\mathrm{R}}-2 \pi \delta\left(p^{2}\right) P_{\mathrm{L}} \not P_{\mathrm{R}}\left[\vartheta\left(p_{0}\right) f_{\ell}(\mathbf{p})+\vartheta\left(-p_{0}\right) \bar{f}_{\ell}(-\mathbf{p})\right], \\
& \mathrm{i} S_{\ell}^{\bar{T}}(p)=-P_{\mathrm{L}} \frac{\mathrm{i} \not p}{p^{2}-\mathrm{i} \varepsilon} P_{\mathrm{R}}-2 \pi \delta\left(p^{2}\right) P_{\mathrm{L}} \not p P_{\mathrm{R}}\left[\vartheta\left(p_{0}\right) f_{\ell}(\mathbf{p})+\vartheta\left(-p_{0}\right) \bar{f}_{\ell}(-\mathbf{p})\right] .
\end{aligned}
$$

Due to the unbroken $\mathrm{SU}(2)_{\mathrm{L}}$ symmetry, we suppress the indices representing the lepton doublet. In principle, the propagators are diagonal matrices

$$
S_{\ell}^{\mathrm{SU}(2)}(u, v)=\delta_{A B} S_{\ell}(u, v), \quad A, B=1,2 .
$$

Whenever these propagators appear within the integrals for the source for the asymmetry $S$ or the singlet neutrino self-energy $\mathbb{L}_{N}$, it is a sufficient approximation to take for $f_{\ell}(\mathbf{p})$ and $\bar{f}_{\ell}(\mathbf{p})$ the equilibrium Fermi-Dirac distribution with zero chemical potential. Only in the washout term $W$, Eq. (97), one should correct this by introducing a lepton chemical potential, in order to describe a charged distribution in kinetic equilibrium $c f$. Eq. (98).

The propagators for the Higgs fields are

$$
\begin{aligned}
& \mathrm{i} \Delta_{\phi}^{<}(p)=2 \pi \delta\left(p^{2}\right)\left[\vartheta\left(p_{0}\right) f_{\phi}(\mathbf{p})+\vartheta\left(-p_{0}\right)\left(1+\bar{f}_{\phi}(-\mathbf{p})\right)\right], \\
& \mathrm{i} \Delta_{\phi}^{>}(p)=2 \pi \delta\left(p^{2}\right)\left[\vartheta\left(p_{0}\right)\left(1+f_{\phi}(\mathbf{p})\right)+\vartheta\left(-p_{0}\right) \bar{f}_{\phi}(-\mathbf{p})\right], \\
& \mathrm{i} \Delta_{\phi}^{T}(p)=\frac{\mathrm{i}}{p^{2}+\mathrm{i} \varepsilon}+2 \pi \delta\left(p^{2}\right)\left[\vartheta\left(p_{0}\right) f_{\phi}(\mathbf{p})+\vartheta\left(-p_{0}\right) \bar{f}_{\phi}(-\mathbf{p})\right], \\
& \mathrm{i} \Delta_{\phi}^{\bar{T}}(p)=-\frac{\mathrm{i}}{p^{2}-\mathrm{i} \varepsilon}+2 \pi \delta\left(p^{2}\right)\left[\vartheta\left(p_{0}\right) f_{\phi}(\mathbf{p})+\vartheta\left(-p_{0}\right) \bar{f}_{\phi}(-\mathbf{p})\right] .
\end{aligned}
$$


As for the leptons, it is understood that

$$
\Delta_{\phi A B}^{\mathrm{SU}(2)}(u, v)=\delta_{A B} \Delta_{\phi}(u, v) \quad A, B=1,2 .
$$

Again, we can substitute equilibrium distributions in the collision integrals. When taking account of spectator effects, one should also assign chemical potentials to the Higgs field and the remaining Standard Model degrees of freedom.

When assuming thermal equilibrium distributions and vanishing chemical potentials, the Kubo-Martin-Schwinger (KMS) relations are

$$
\begin{aligned}
& \mathrm{i} S_{\ell}^{>}(k)=-\mathrm{e}^{k^{0} / T} \mathrm{i} S_{\ell}^{<}(k), \\
& \mathrm{i} \Delta_{\phi}^{>}(k)=\mathrm{e}^{k^{0} / T} \mathrm{i} \Delta_{\phi}^{<}(k) .
\end{aligned}
$$

Notice that the according relations also hold for the fermionic and scalar equilibrium self-energies.

\section{Self-energies from the 2PI Effective Action}

The two-loop contribution 2PI effective action arising from the Yukawa interaction in Eq. (44) is given by

$$
\Gamma_{2}^{(2)}=-g_{w} Y_{i}^{*} Y_{j} \sum_{a, b= \pm} a b \int d^{4} x d^{4} y \operatorname{tr}\left[P_{\mathrm{R}} S_{i j}^{a b}(x, y) P_{\mathrm{L}} S_{\ell}^{b a}(y, x)\right] \Delta_{\phi}^{b a}(y, x),
$$

where $a, b= \pm$ denote the CTP indices, the trace is over Dirac indices and sum over neutrino flavours $i$ is understood. The one-loop neutrino self-energy is then given by

$$
\begin{aligned}
\mathrm{i} \Sigma_{N i j}^{a b}(x, y) & =a b \frac{\delta \Gamma_{2}}{\delta S_{j i}^{b a}(y, x)} \\
& =g_{w}\left(Y_{i} Y_{j}^{*} P_{\mathrm{L}} \mathrm{i} S_{\ell}^{a b}(x, y) P_{\mathrm{R}} \mathrm{i} \Delta_{\phi}^{a b}(x, y)+Y_{i}^{*} Y_{j} C P_{\mathrm{R}} \mathrm{i} S_{\ell}^{b a t}(y, x) C^{\dagger} P_{\mathrm{L}} \mathrm{i} \Delta_{\phi}^{b a}(y, x)\right) .
\end{aligned}
$$

Two contributions arise from the functional derivative, because $S^{a b}$ and $S^{b a}$ are not independent but related by the Majorana symmetry: $S_{i j}^{a b}(x, y)=C S_{j i}^{b a}(y, x) C^{\dagger}$. Similarly, using $g_{w} S_{\ell}=\delta_{A B} S_{\ell A B}^{\mathrm{SU}(2)}$ in Eq. (125) we get for the the lepton self-energy

$$
\begin{aligned}
\mathrm{i} \Sigma_{\ell A B}^{\mathrm{SU}(2) a b}(x, y) & =a b \frac{\delta \Gamma_{2}}{\delta S_{\ell B A}^{\mathrm{SU}(2) b a}(y, x)}=Y_{i}^{*} Y_{j} P_{\mathrm{R}} \mathrm{i} S_{i j}^{a b}(u, v) P_{\mathrm{L}} \mathrm{i} \Delta_{\phi}^{b a}(v, u) \delta_{A B} \\
& \equiv \mathrm{i} \Sigma_{\ell}^{a b}(x, y) \delta_{A B} .
\end{aligned}
$$




\section{E Explicit Form of the Helicity Block-Diagonal De- composition}

In order quickly see the relation of the functions $g_{a h}$ with the vector, scalar, pseudoscalar and pseudovector densities, it is useful to recast Eq. (56) as

$$
\begin{aligned}
\mathrm{i} S_{N}=\sum_{h= \pm}-\frac{1}{4}\left[\left(\begin{array}{ll}
0 & \mathbb{1} \\
\mathbb{1} & 0
\end{array}\right)+h \hat{k}^{i}\left(\begin{array}{cc}
0 & \sigma^{i} \\
\sigma^{i} & 0
\end{array}\right)\right] g_{0 h} \\
-\frac{1}{4}\left[\left(\begin{array}{ll}
\mathbb{1} & 0 \\
0 & \mathbb{1}
\end{array}\right)+h \hat{k}^{i}\left(\begin{array}{cc}
\sigma^{i} & 0 \\
0 & \sigma^{i}
\end{array}\right)\right] g_{1 h} \\
-\frac{1}{4}\left[\left(\begin{array}{cc}
\mathrm{i} \mathbb{1} & 0 \\
0 & -\mathrm{i} \mathbb{1}
\end{array}\right)+h \hat{k}^{i}\left(\begin{array}{cc}
\mathrm{i} \sigma^{i} & 0 \\
0 & -\mathrm{i} \sigma^{i}
\end{array}\right)\right] g_{2 h} \\
-\frac{1}{4}\left[\left(\begin{array}{cc}
0 & -\mathbb{1} \\
\mathbb{1} & 0
\end{array}\right)+h \hat{k}^{i}\left(\begin{array}{cc}
0 & -\sigma^{i} \\
\sigma^{i} & 0
\end{array}\right)\right] g_{3 h} .
\end{aligned}
$$

\section{References}

[1] M. Fukugita and T. Yanagida, "Baryogenesis Without Grand Unification," Phys. Lett. B 174 (1986) 45.

[2] M. A. Luty, "Baryogenesis via leptogenesis," Phys. Rev. D 45 (1992) 455.

[3] L. Covi, E. Roulet and F. Vissani, "CP violating decays in leptogenesis scenarios," Phys. Lett. B 384 (1996) 169 [hep-ph/9605319].

[4] W. Buchmuller and M. Plumacher, "CP asymmetry in Majorana neutrino decays," Phys. Lett. B 431 (1998) 354 [hep-ph/9710460].

[5] E. W. Kolb and S. Wolfram, "Baryon Number Generation in the Early Universe," Nucl. Phys. B 172 (1980) 224 [Erratum-ibid. B 195 (1982) 542].

[6] M. Flanz, E. A. Paschos, U. Sarkar and J. Weiss, "Baryogenesis through mixing of heavy Majorana neutrinos," Phys. Lett. B 389 (1996) 693 [hep-ph/9607310].

[7] A. Pilaftsis, "Resonant CP violation induced by particle mixing in transition amplitudes," Nucl. Phys. B 504 (1997) 61 hep-ph/9702393].

[8] A. Pilaftsis, "CP violation and baryogenesis due to heavy Majorana neutrinos," Phys. Rev. D 56 (1997) 5431 hep-ph/9707235.

[9] A. Pilaftsis and T. E. J. Underwood, "Resonant leptogenesis," Nucl. Phys. B 692 (2004) 303 hep-ph/0309342. 
[10] A. Pilaftsis and T. E. J. Underwood, "Electroweak-scale resonant leptogenesis," Phys. Rev. D 72 (2005) 113001 [hep-ph/0506107].

[11] A.Yu. Ignat'ev, V.A. Kuz'min and M.E. Shaposhnikov, "The origin of baryon asymmetry in the universe," JETP Lett. 30 (1979) 688.

[12] F. J. Botella and J. Roldan, "The epsilon type contribution to baryon asymmetry from colored Higgs triplets," Phys. Rev. D 44 (1991) 966.

[13] J. Liu and G. Segre, "Reexamination of generation of baryon and lepton number asymmetries by heavy particle decay," Phys. Rev. D 48 (1993) 4609 hep-ph/9304241.

[14] J. S. Schwinger, "Brownian motion of a quantum oscillator," J. Math. Phys. 2 (1961) 407.

[15] L. V. Keldysh, "Diagram technique for nonequilibrium processes," Zh. Eksp. Teor. Fiz. 47 (1964) 1515 [Sov. Phys. JETP 20 (1965) 1018].

[16] K. -c. Chou, Z. -b. Su, B. -l. Hao and L. Yu, "Equilibrium and Nonequilibrium Formalisms Made Unified," Phys. Rept. 118 (1985) 1.

[17] E. Calzetta and B. L. Hu, "Nonequilibrium Quantum Fields: Closed Time Path Effective Action, Wigner Function and Boltzmann Equation," Phys. Rev. D 37 (1988) 2878.

[18] R. E. Cutkosky, "Singularities and discontinuities of Feynman amplitudes," J. Math. Phys. 1 (1960) 429.

[19] A. D. Dolgov, "Neutrinos in the Early Universe," Sov. J. Nucl. Phys. 33 (1981) 700 [Yad. Fiz. 33 (1981) 1309].

[20] R. Barbieri and A. Dolgov, "Neutrino oscillations in the early universe," Nucl. Phys. B 349 (1991) 743.

[21] K. Enqvist, K. Kainulainen and J. Maalampi, "Refraction And Oscillations Of Neutrinos In The Early Universe," Nucl. Phys. B 349 (1991) 754.

[22] K. Enqvist, K. Kainulainen and M. J. Thomson, "Stringent cosmological bounds on inert neutrino mixing," Nucl. Phys. B 373 (1992) 498.

[23] G. Sigl and G. Raffelt, "General kinetic description of relativistic mixed neutrinos," Nucl. Phys. B 406 (1993) 423.

[24] E. K. Akhmedov, V. A. Rubakov and A. Y. .Smirnov, "Baryogenesis via neutrino oscillations," Phys. Rev. Lett. 81 (1998) 1359 hep-ph/9803255. 
[25] T. Asaka and M. Shaposhnikov, "The nuMSM, dark matter and baryon asymmetry of the universe," Phys. Lett. B 620 (2005) 17 [hep-ph/0505013].

[26] J. -S. Gagnon and M. Shaposhnikov, "Baryon Asymmetry of the Universe without Boltzmann or Kadanoff-Baym equations," Phys. Rev. D 83 (2011) 065021 arXiv:1012.1126 [hep-ph]].

[27] W. Buchmuller and S. Fredenhagen, "Quantum mechanics of baryogenesis," Phys. Lett. B 483, 217 (2000) hep-ph/0004145.

[28] A. De Simone and A. Riotto, "Quantum Boltzmann Equations and Leptogenesis," JCAP 0708 (2007) 002 hep-ph/0703175].

[29] M. Garny, A. Hohenegger, A. Kartavtsev and M. Lindner, "Systematic approach to leptogenesis in nonequilibrium QFT: vertex contribution to the CP-violating parameter," Phys. Rev. D 80 (2009) 125027 [arXiv:0909.1559 [hep-ph]].

[30] M. Garny, A. Hohenegger, A. Kartavtsev and M. Lindner, "Systematic approach to leptogenesis in nonequilibrium QFT: self-energy contribution to the CP-violating parameter," Phys. Rev. D 81 (2010) 085027 [arXiv:0911.4122 [hep-ph]].

[31] A. Anisimov, W. Buchmüller, M. Drewes and S. Mendizabal, "Leptogenesis from Quantum Interference in a Thermal Bath," Phys. Rev. Lett. 104 (2010) 121102 arXiv:1001.3856 [hep-ph]].

[32] M. Garny, A. Hohenegger, A. Kartavtsev, "Medium corrections to the CP-violating parameter in leptogenesis," Phys. Rev. D81 (2010) 085028. arXiv:1002.0331 [hep$\mathrm{ph}]]$.

[33] M. Beneke, B. Garbrecht, M. Herranen and P. Schwaller, "Finite Number Density Corrections to Leptogenesis," Nucl. Phys. B 838 (2010) 1 arXiv:1002.1326 [hep$\mathrm{ph}]]$.

[34] M. Beneke, B. Garbrecht, C. Fidler, M. Herranen and P. Schwaller, "Flavoured Leptogenesis in the CTP Formalism," Nucl. Phys. B 843 (2011) 177 arXiv:1007.4783 [hep-ph]].

[35] M. Garny, A. Hohenegger and A. Kartavtsev, "Quantum corrections to leptogenesis from the gradient expansion," arXiv:1005.5385 [hep-ph].

[36] B. Garbrecht, "Leptogenesis: The Other Cuts," Nucl. Phys. B847 (2011) 350-366. arXiv:1011.3122 [hep-ph]].

[37] A. Anisimov, W. Buchmuller, M. Drewes and S. Mendizabal, "Quantum Leptogenesis I," Annals Phys. 326 (2011) 1998 arXiv:1012.5821 [hep-ph]].

[38] T. Asaka, M. Laine and M. Shaposhnikov, "On the hadronic contribution to sterile neutrino production," JHEP 0606 (2006) 053 hep-ph/0605209]. 
[39] C. P. Kiessig, M. Plumacher and M. H. Thoma, "Decay of a Yukawa fermion at finite temperature and applications to leptogenesis," Phys. Rev. D 82 (2010) 036007 arXiv:1003.3016 [hep-ph]].

[40] A. Anisimov, D. Besak and D. Bodeker, "Thermal production of relativistic Majorana neutrinos: Strong enhancement by multiple soft scattering," JCAP 1103 (2011) 042 [arXiv:1012.3784 [hep-ph]].

[41] A. Salvio, P. Lodone and A. Strumia, "Towards leptogenesis at NLO: the righthanded neutrino interaction rate," JHEP 1108 (2011) 116 arXiv:1106.2814 [hep$\mathrm{ph}]]$.

[42] C. Kiessig and M. Plumacher, "Hard-Thermal-Loop Corrections in Leptogenesis I: CP-Asymmetries," arXiv:1111.1231 [hep-ph].

[43] C. Kiessig and M. Plumacher, "Hard-Thermal-Loop Corrections in Leptogenesis II: Solving the Boltzmann Equations," arXiv:1111.1235 [hep-ph].

[44] M. Laine and Y. Schroder, "Thermal right-handed neutrino production rate in the non-relativistic regime," arXiv:1112.1205 [hep-ph].

[45] J. Liu and G. Segre, "Unstable particle mixing and CP violation in weak decays," Phys. Rev. D 49 (1994) 1342 hep-ph/9310248.

[46] L. Covi and E. Roulet, "Baryogenesis from mixed particle decays," Phys. Lett. B 399 (1997) 113 hep-ph/9611425.

[47] A. Anisimov, A. Broncano and M. Plumacher, "The CP-asymmetry in resonant leptogenesis," Nucl. Phys. B 737 (2006) 176 [hep-ph/0511248].

[48] C. Greiner and S. Leupold, "Stochastic interpretation of Kadanoff-Baym equations and their relation to Langevin processes," Annals Phys. 270 (1998) 328 hep-ph/9802312.

[49] C. Greiner and S. Leupold, "Interpretation and resolution of pinch singularities in nonequilibrium quantum field theory," Eur. Phys. J. C 8 (1999) 517 hep-ph/9804239].

[50] B. Garbrecht and M. Garny, "Finite Width in out-of-Equilibrium Propagators and Kinetic Theory," Annals Phys. 327 (2012) 914 arXiv:1108.3688 [hep-ph]].

[51] T. Prokopec, M. G. Schmidt and S. Weinstock, "Transport equations for chiral fermions to order h-bar and electroweak baryogenesis," Annals Phys. 314 (2004) 208 arXiv:hep-ph/0312110.

[52] T. Prokopec, M. G. Schmidt and S. Weinstock, "Transport equations for chiral fermions to order h-bar and electroweak baryogenesis. II," Annals Phys. 314 (2004) 267 arXiv:hep-ph/0406140]. 
[53] V. Cirigliano, C. Lee, M. J. Ramsey-Musolf, S. Tulin, "Flavored Quantum Boltzmann Equations," Phys. Rev. D81 (2010) 103503. arXiv:0912.3523 [hep-ph]].

[54] V. Cirigliano, C. Lee, S. Tulin, "Resonant Flavor Oscillations in Electroweak Baryogenesis," arXiv:1106.0747 [hep-ph]].

[55] M. Herranen, K. Kainulainen and P. M. Rahkila, "Towards a kinetic theory for fermions with quantum coherence," Nucl. Phys. B 810 (2009) 389 [arXiv:0807.1415 [hep-ph]].

[56] M. Herranen, K. Kainulainen and P. M. Rahkila, "Quantum kinetic theory for fermions in temporally varying backgrounds," JHEP 0809 (2008) 032 arXiv:0807.1435 [hep-ph]].

[57] M. Herranen, K. Kainulainen and P. M. Rahkila, "Kinetic theory for scalar fields with nonlocal quantum coherence," JHEP 0905 (2009) 119 arXiv:0812.4029v2 [hep$\mathrm{ph}]]$.

[58] M. Herranen, K. Kainulainen and P. M. Rahkila, "Coherent quasiparticle approximation cQPA and nonlocal coherence," J. Phys. Conf. Ser. 220 (2010) 012007 arXiv:0912.2490 [hep-ph]].

[59] M. Herranen, "Quantum kinetic theory with nonlocal coherence," PhD Thesis, University of Jyväskylä Res. Rep. 3/2009 [arXiv:0906.3136 [hep-ph]].

[60] M. Herranen, K. Kainulainen and P. M. Rahkila, "Coherent quantum Boltzmann equations from cQPA," JHEP 1012 (2010) 072 [arXiv:1006.1929 [hep-ph]].

[61] C. Fidler, M. Herranen, K. Kainulainen and P. M. Rahkila, "Flavoured quantum Boltzmann equations from cQPA," arXiv:1108.2309 [hep-ph].

[62] M. Herranen, K. Kainulainen and P. M. Rahkila, "Flavour-coherent propagators and Feynman rules: Covariant cQPA formulation," arXiv:1108.2371 [hep-ph].

[63] T. Konstandin, T. Prokopec, M. G. Schmidt and M. Seco, "MSSM electroweak baryogenesis and flavour mixing in transport equations," Nucl. Phys. B 738 (2006) 1 [arXiv:hep-ph/0505103].

[64] Y. Grossman, T. Kashti, Y. Nir and E. Roulet, "Leptogenesis from supersymmetry breaking," Phys. Rev. Lett. 91 (2003) 251801 [hep-ph/0307081].

[65] G. D'Ambrosio, G. F. Giudice and M. Raidal, "Soft leptogenesis," Phys. Lett. B $\mathbf{5 7 5}$ (2003) 75 [hep-ph/0308031].

[66] C. S. Fong, M. C. Gonzalez-Garcia and E. Nardi, "Leptogenesis from Soft Supersymmetry Breaking (Soft Leptogenesis)," Int. J. Mod. Phys. A 26 (2011) 3491 arXiv:1107.5312 [hep-ph]]. 
[67] T. Hahn, "Routines for the diagonalization of complex matrices," physics/0607103.

[68] J. Busch, "Kinetic Equations for Leptogenesis in the 2PI-Formalism," Diploma Thesis, RWTH Aachen 2011.

[69] S. Blanchet, T. Hambye and F. -X. Josse-Michaux, "Reconciling leptogenesis with observable mu $\rightarrow$ e gamma rates," JHEP 1004 (2010) 023 arXiv:0912.3153 [hep$\mathrm{ph}]$.

[70] K. Kainulainen, T. Prokopec, M. G. Schmidt and S. Weinstock, "First principle derivation of semiclassical force for electroweak baryogenesis," JHEP 0106 (2001) 031 [hep-ph/0105295].

[71] B. Garbrecht, T. Prokopec and M. G. Schmidt, "Particle number in kinetic theory," Eur. Phys. J. C 38 (2004) 135 [hep-th/0211219].

[72] M. Garny, A. Kartavtsev and A. Hohenegger, "Leptogenesis from first principles in the resonant regime," arXiv:1112.6428 [hep-ph].

[73] T. Asaka, S. Eijima and H. Ishida, "Kinetic Equations for Baryogenesis via Sterile Neutrino Oscillation," arXiv:1112.5565 [hep-ph]. 